\title{
Streamwise vortex structure in plane mixing layers
}

\author{
By L. P. BERNAL† AND A. ROSHKO \\ Graduate Aeronautical Laboratories, California Institute of Technology, \\ Pasadena, CA 91125, USA
}

(Received 9 September 1985 and in revised form 13 March 1986)

The development of three-dimensional motions in a plane mixing layer was investigated experimentally. It is shown that superimposed on the primary, spanwise vortex structure there is a secondary, steamwise vortex structure. Three aspects of this secondary structure were studied. First, the spanwise vortex instability that generates the secondary structure was characterized by measurements of the critical Reynolds number and the spanwise wavelength at several flow conditions. While the critical Reynolds number was found to depend on the velocity ratio, density ratio and initial shear-layer-profile shape, the mean normalized wavelength is independent of these parameters. Secondly, flow visualization in water was used to obtain cross-sectional views of the secondary structure associated with the streamwise counter-rotating vortices. A model is proposed in which those vortices are part of a single vortex line winding back and forth between the high-speed side of a primary vortex and the low-speed side of the following one. Finally, the effect of the secondary structure on the spanwise concentration field was measured in a helium-nitrogen mixing layer. The spatial organization of the secondary structure produces a well-defined spanwise entrainment pattern in which fluid from each stream is preferentially entrained at different spanwise locations. These measurements show that the spanwise scale of the secondary structure increases with downstream distance.

\section{Introduction}

The turbulent plane mixing layer (figure 1) has been the subject of several investigations in this laboratory beginning with the finding of Brown \& Roshko $(1971,1974)$ that spanwise-organized, large vortical structures exist in this flow up to high values of Reynolds number. On the pictures obtained it was evident that the flow also contains smaller-scale structures, which form no particular patterns on edge views of the layer but appear as distinctive streamwise streaks in plan view. An example at relatively low Reynolds number appeared in the paper of Brown \& Roshko (1974) and a more complete set in the thesis of Konrad (1976), from which an example at high Reynolds number is reproduced in figure 9. Those pictures were obtained in gas flows, using shadowgraph techniques. In water, using chemical-dye techniques Breidenthal $(1978,1981)$ also observed streamwise streaks and noted that they appeared to result from the stretching of a spanwise instability wave ('wiggle') which forms early in the flow.

It was speculated that the streaks delineate streamwise vortices which contribute

$\dagger$ Present Address: Department of Aerospace Engineering, The University of Michigan, Ann Arbor, Michigan 48109-2140, USA. 


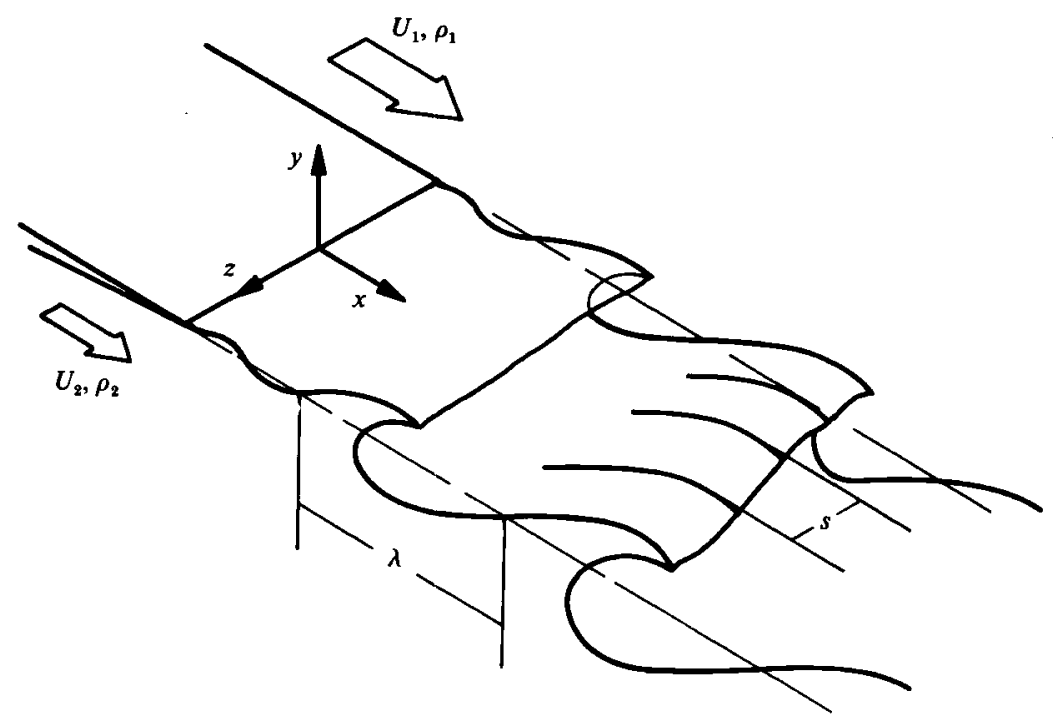

Figure 1. The plane mixing layer.

in some way to the increased amount of mixing that occurs in the mixing transition region of the shear layer, first identified by Konrad and further investigated by Breidenthal. The primary, spanwise vortices and their amalgamations occur on both sides of the mixing transition, but the transition is characterized by the appearance, on its high-Reynolds-number or downstream side, of small-scale, three-dimensional structures (as seen on flow-visualization pictures, e.g. figure 9) and by an increased amount of mixing (as exhibited by the extent of chemical reaction between reactants carried by the two streams). Visual observation of streamwise vortices had previously been reported by Bradshaw (1966) and Miksad (1972). The streaky features found in the concentration field have also been observed on velocity-field measurements by Jimenez (1983). Both Jimenez (1983) and Hussain (1983) proposed models of the three-dimensional structure in plane mixing layers similar to the one first described in Bernal (1981) and discussed here. More recently Lasheras, Cho \& Maxworthy (1986) studied experimentally the initial development of three-dimensionality in plane mixing layers.

The purpose of the present investigation was to obtain further information about the nature of the motions associated with the streamwise streaks, their relation to development of three-dimensionality in the mixing layer and their contributions to mixing and growth of the layer.

Relevant to this is the role of the primary, spanwise-organized vortical structures in the growth of the mixing layer. Those vortices are convected downstream during lifetimes that are terminated when they interact or amalgamate with neighbouring vortices to form larger ones. A form of interaction, the pairing interaction, in which two adjacent vortices rotate around each other until they amalgamate, was described by Winant \& Browand (1974) at low and moderate Reynolds numbers. This form of amalgamation and similar ones involving more than two vortices have also been observed at high Reynolds numbers (see Roshko 1976; Dimotakis \& Brown 1976; Hernan \& Jimenez 1982). The amalgamation of more than two vortices has been studied by Ho \& Huang (1982) in a forced mixing layer. Another form of interaction, the tearing process, that is the shearing of a vortex between its neighbours, is also 
possible and has been observed by Winant \& Browand (1974), Dimotakis \& Brown (1976) and ourselves as reported by Hernan \& Jimenez (1982).

Winant \& Browand proposed that the growth of the mixing layer is primarily due to amalgamation processes but Bernal (1981) and Hernan \& Jimenez, who studied motion pictures obtained at much higher Reynolds numbers than those in the experiments of Winant \& Browand, found that considerable (possibly principal) growth occurs in individual vortices during their lifetime between coalescences. This observation is consistent with the important point made by Moore \& Saffman (1975) that direct growth of the vortices by turbulent entrainment must play an essential role. Their argument is based on the need to ingest irrotational fluid into the vortices so that the necessary decrease in vorticity can occur as the mixing layer grows downstream. At low Reynolds numbers this ingestion could occur by viscous diffusion, as in the flows calculated by Patnaik, Sherman \& Corcos (1976), but at high Reynolds numbers it would have to occur by small-scale turbulent mixing as suggested by Moore \& Saffman.

Other forms of interaction, non-uniform pairing events, were observed by Chandrsuda et al. (1978). These observations as well as cross-correlation measurements between velocity probes deployed along the span of the mixing layer led Chandrsuda et al. to question the persistence of the spanwise organized structures in the fully developed flow. However, Wygnanski et al. (1979) reported similar correlation measurements obtained both at the edge of the layer and at its centre. The results at the edge of the layer revealed the presence of spanwise correlated structures, while those at the centre showed a much shorter spanwise characteristic length associated with the smaller scale structure embedded within the large-scale vortices. Browand \& Troutt (1980) measured somewhat lower cross-correlation coefficients than Wygnanski et al. at the edge of the layer, yet their instantaneous realizations of the velocity field showed good spanwise correlation of the velocity fluctuations. Browand \& Troutt argued that spanwise non-uniformities would be amplified during amalgamations, thus degrading the cross-correlation coefficient even though the large-scale structure and its dynamics are by and large two-dimensional. It might also be argued that smaller-scale, secondary instabilities such as those associated with the streamwise vortices would contribute to degradation of spanwise correlation, especially if their locations in space are fluctuating. The velocities associated with these can be quite high, as indicated by the experiments of Katz \& O'Hern (1983) who found that, in mixing layers in water, cavitation occurs first in the streamwise vortices. These views of the primary vortex structure and processes in a mixing layer illustrate the need to consider the effects of what might be called residual turbulence on those processes, i.e. the effects of secondary and possibly higher-order instabilities (considering the spanwise-organized vortices to be the structures resulting from the primary instability).

The present experiments were conducted in the gas mixing-layer facility used by Brown \& Roshko (1971) and in Breidenthal's (1981) water facility. The facilities and experimental techniques are briefly described in \$2. A more complete description is given in Bernal (1981). In $\$ 3$ we study the origin of the structure associated with the streamwise streak pattern. Flow visualization of mixing layers between helium and nitrogen streams was used to characterize the effect of the apparatus and flow parameters on the streak pattern early in its history. The question of the vortical nature of the structure is addressed next, in $\$ 4$. Flow visualization of a cross-sectional plane in the water facility provided direct evidence of the streamwise vortices. Analysis of a flow-visualization motion picture is used to obtain the topology of the 


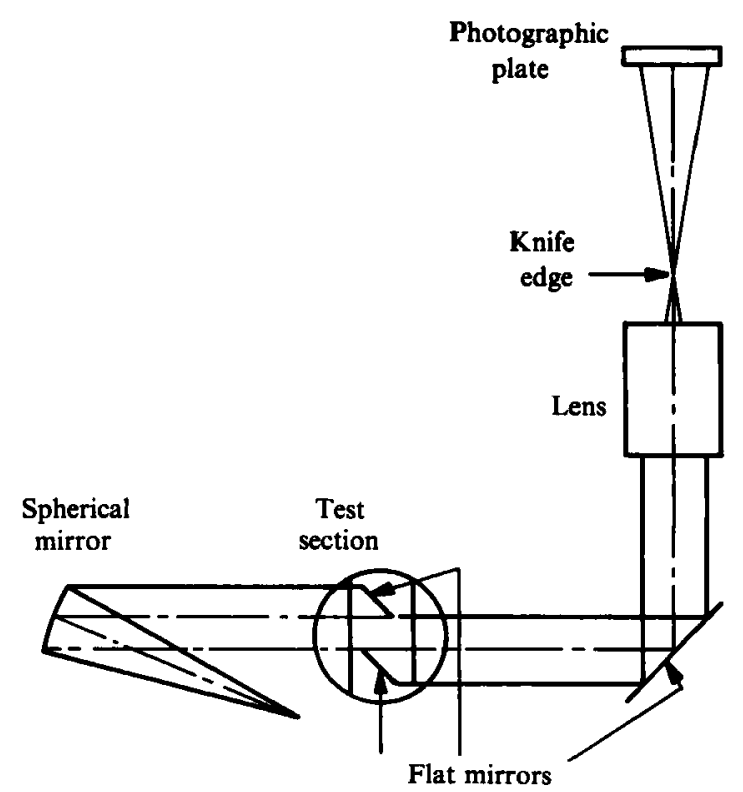

Figure 2. Schlieren optical system.

streamwise vortices and their relation to the primary spanwise vortices. Finally in $\$ 5$ the effects of the streamwise vortex structure on the concentration field are established from concentration measurements in the mixing layer between helium and nitrogen streams. Some implications of these results are discussed in $\S 6$.

\section{Flow facilities and instrumentation}

\subsection{Gas facility}

The gas mixing-layer facility is a two-channel pessurized blowdown tunnel. The gases are stored in high-pressure bottles, eight bottles per channel. Two independent lines, which include a pressure regulator, a metering valve and a remotely operated ball valve, provide an adjustable mass flow for each channel. The working section is enclosed in a high-pressure vessel, whose pressure is maintained at the required level by a regulator.

In each channel, the gas flow enters a stagnation section which contains several turbulence-reducing elements, namely a $0.64 \mathrm{~cm}$ thick foam, a perforated plate with $44 \%$ open-area ratio, a $4 \mathrm{~cm}$ long honeycomb section with $0.2 \mathrm{~cm}$ cell size and a fine mesh screen with $50 \%$ open-area ratio. The stagnation section is followed by a $4: 1$ two-dimensional contraction. The resulting free-stream turbulence level is below $0.5 \%$. The two channels are separated by a partition. Two interchangeable partitions of different length were used. The standard partition terminates at the entrance to the test section. A second partition which was $2.5 \mathrm{~cm}$ longer was used on some tests, as indicated in $\$ 3$. The effective origin of the boundary layers on the partitions was estimated as $L_{\mathrm{LB}}=3.0 \mathrm{~cm}$ and $5.5 \mathrm{~cm}$ for the standard and extended partitions respectively. In all test conditions the boundary layers at the end of the partition were laminar.

The test section is rectangular in cross-section, $5 \mathrm{~cm}$ high by $10 \mathrm{~cm}$ wide. At the entrance, each stream occupies one-half of the cross-section, i.e. $10 \mathrm{~cm} \times 2.5 \mathrm{~cm}$, the larger dimension being spanwise. The top and bottom walls, those parallel to the 
mixing layer, are adjustable so that the pressure gradient in the flow direction can be minimized. The length of the test section is $25 \mathrm{~cm}$, but only the initial $11 \mathrm{~cm}$ was used in these measurements.

Shadowgraph and Schlieren flow-visualization techniques were used in the experiments. The optical system is sketched in figure 2. Simultaneous side and plan views were obtained by deflecting a portion of the incoming beam perpendicular to the plane of the layer, as shown in the figure. In the Schlieren system the knife edge was positioned parallel to the flow direction, thus the photographic density on the film is proportional to the flow density gradient across the layer in the side view and along the span in the plan view, respectively. A stroboscopic light source was used for the Schlieren pictures. Time exposures were obtained by strobing the unit at $100 \mathrm{~Hz}$ for 2 s. A similar system was used for the shadowgraph motion pictures (Bernal 1981).

The molar fraction of helium $C$ was measured with concentration probes similar to those developed by Brown \& Rebollo (1972). The probes were calibrated regularly against a mixture of known composition to an accuracy of \pm 0.01 . Power-spectrum measurements of the concentration fluctuation showed no appreciable energy content above $15 \mathrm{kHz}$. At the velocities used, this value corresponds to a spatial resolution of $0.02 \mathrm{~cm}$.

Two concentration probes and one Pitot-static probe deployed at fixed positions along the span were used in most experiments. During each experiment the set of three probes was traversed normal to the shear layer, stopping at 21 points within the layer. The position accuracy was $0.02 \mathrm{~cm}$. At each point the probes' output was digitized sequentially at a combined rate of $86 \mathrm{kHz}$ for $0.18 \mathrm{~s}$ and stored on digital tape. From these data the mean and r.m.s. values of the concentration fluctuation were computed at every measurement point. This averaging time is the longest consistent with the total test time of $5 \mathrm{~s}$ required to minimize temperature changes in the supply bottles. It corresponds to passage of 30 spanwise vortices at $x=9 \mathrm{~cm}$.

\subsection{Water facility}

The mixing-layer facility designed by Breidenthal (1981) was used for flowvisualization tests in water. This facility is a gravity-driven apparatus in which each stream is supplied from a different reservoir. The free-stream turbulence intensity was below $0.5 \%$. At the maximum velocity used in this investigation $(100 \mathrm{~cm} / \mathrm{s})$ the boundary layers on the splitter-plate were still laminar. For additional details on this facility the reader is referred to Breidenthal $(1978,1981)$.

Laser-induced fluorescence was used to visualize the flow (Dewey, 1976; Dimotakis, Miake-Lye \& Papantoniou 1983). Fluorescein dye was added to the low-speed fluid. The test section was illuminated with a plane sheet of light less than $0.05 \mathrm{~cm}$ thick, which was formed from an argon-ion laser beam by means of a spherical and cylindrical lens. Dye within the plane of illumination was rendered visible by its fluorescence, and was recorded by conventional photographic techniques. The plane of illumination could be located at any position or orientation relative to the flow. However, cross-sectional views ( $y, z$-plane) had to be viewed and recorded at an angle of approximately $30^{\circ}$ with respect to the downstream direction. This constraint resulted in some geometrical distortion and reduced resolution of $(y, z)$-cross-section pictures.

\section{Origin of the streamwise streaks}

Quantitative data on the onset of the streamwise vortices were obtained from flow-visualization Schlieren pictures of the mixing layer between helium and nitrogen streams. Figure 3 is an instantaneous visualization (1-2 $\mu$ s) of the layer obtained at 


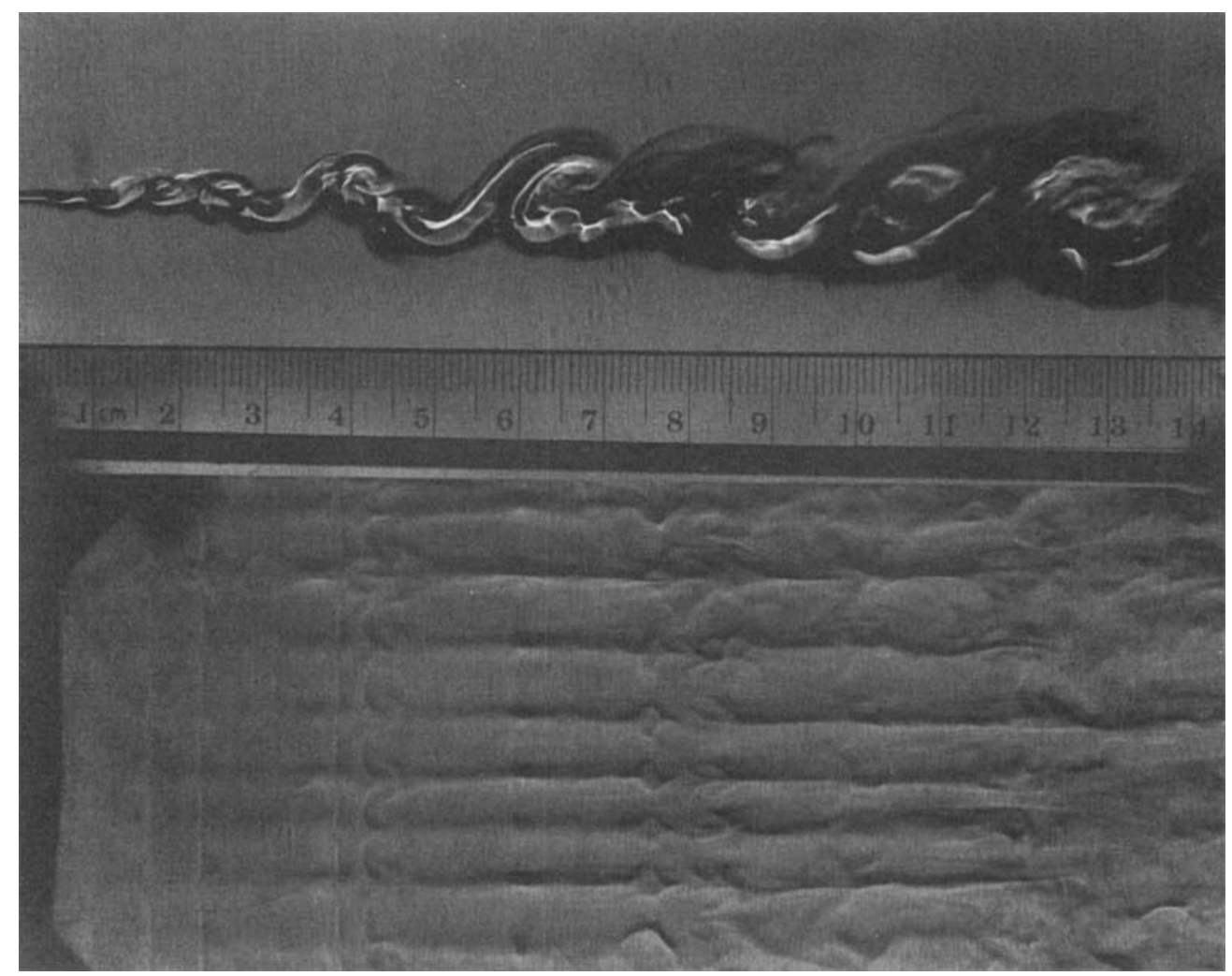

FIaURe 3. Instantaneous Schlieren picture of the helium-nitrogen mixing layer. $U_{1}=496 \mathrm{~cm} / \mathrm{s}, r=0.5, \rho_{2} / \rho_{1}=7, P_{0}=4 \mathrm{~atm}(1-2 \mu \mathrm{s}$ exposure $)$.

a Reynolds number per unit length $\Delta U / \nu_{2}=65000 \mathrm{~cm}^{-1}$, where $\Delta U=U_{1}-U_{2}$ and $\nu_{2}$ is the kinematic viscosity of nitrogen. The streamwise streaks are clearly apparent in the plan-view portion of the picture. Figure 4 shows a time-exposure picture $(2 \mathrm{~s})$ of the mixing layer obtained at the same conditions as figure 3 . A well-defined streamwise streak pattern is also apparent in the time exposure. Furthermore the position of the streamwise streaks in both pictures is the same, implying that there is little fluctuation in their spanwise positions.

The persistence of the streaks at fixed spanwise positions suggests that they could be associated with disturbances originating somewhere upstream in the settling chamber. Indeed, changes in the screens produced changes in their spanwise positions. On the other hand, it is clear that disturbances associated with the streaks are being amplified from their upstream origin until they reach levels at which they become visible on the photographs. The location of this visible threshold amplitude could be shifted upstream or downstream by variation of the Reynolds number or the velocity ratio. These flow changes also resulted in changes of the number of streaks. For example, figure 5 shows an instantaneous picture of the flow obtained at the same experimental conditions as figure 3 except for a change in velocities as indicated $\left(\Delta U / \nu_{2}=27000 \mathrm{~cm}^{-1}\right)$. Figure 6 is a time-exposure picture obtained at the same conditions. There is a considerable change in the streamwise streak pattern compared with the low-Reynolds-number case. Our conclusion from these and other comparisons, discussed below, is that these initial streaks are associated with true 


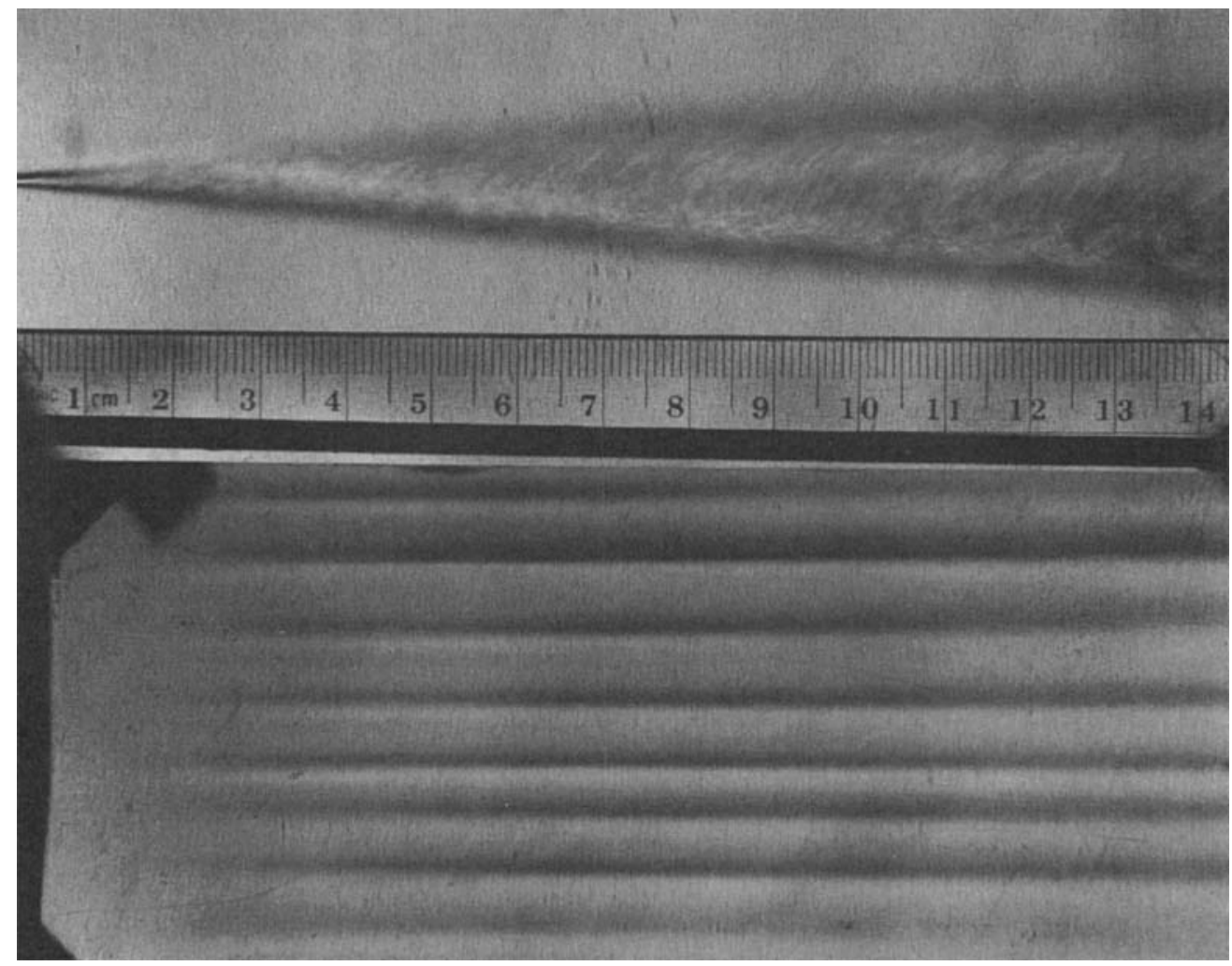

Fiqure 4. Time-exposure Schlieren picture of the helium-nitrogen mixing layer.

Same flow conditions as in figure 3 ( 2 s exposure).

flow instabilities which, of course, originate from the available upstream disturbances, but which respond differently to different flow conditions. In particular we studied the dependence of the mean spacing and initial location of the visible pattern on flow parameters.

The downstream distance to the visual origin of the pattern was characterized by the visual thickness of the layer $\delta_{\mathrm{vi}}$ at that point, measured on the time-exposure pictures. Based on the visual thickness we define the critical Reynolds number $\Delta U \delta_{\mathrm{vi}} / \nu_{2}$. The results of the measurements are summarized in table 1 . The variation of this critical Reynolds number with $\Delta U / U_{\mathrm{c}}$ is presented in figure 7 , where $U_{\mathrm{c}}$ is the convection velocity of the primary vortices. $U_{\mathrm{c}}$ is related to the velocity ratio, $r=U_{2} / U_{1}$, and the density ratio $\rho_{2} / \rho_{1}$, by the expression (Brown 1974)

$$
\frac{\Delta U}{U_{\mathrm{c}}}=\left\{1+\left(\rho_{2} / \rho_{1}\right)^{\frac{1}{2}}\right\} \frac{1-r}{1+r\left(\rho_{2} / \rho_{1}\right)^{\frac{1}{2}}} .
$$

As shown in figure 7, the critical Reynolds number depends on this parameter, changing by a factor of 4 over the range 0.8-2.2 which corresponds to a change in velocity ratio from 0.15 to 0.5 . The increased length of the splitter plate, $L_{\mathrm{BL}}=5.5 \mathrm{~cm}$, results in a corresponding increase of the critical Reynolds number at the same velocity ratio. We believe that this is due mainly to a change of the initial shear-layer-profile shape.

These results for $\rho_{2} / \rho_{1}=7$ can be compared with a local Reynolds number 


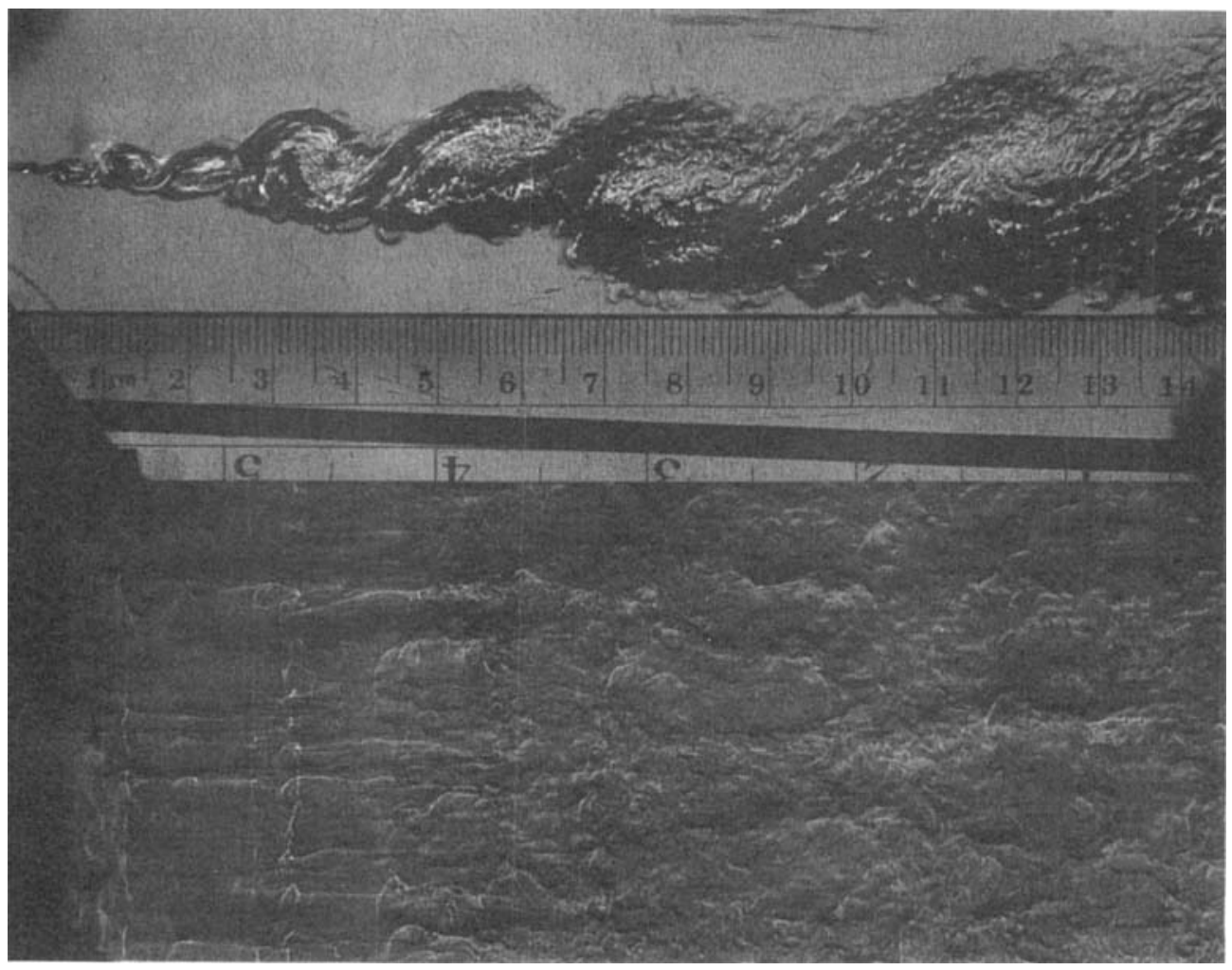

FIGURE 5. Instantaneous Schlieren picture of the helium-nitrogen mixing layer.

$$
U_{1}=1470 \mathrm{~cm} / \mathrm{s}, r=0.3, \rho_{2} / \rho_{1}=7, P_{0}=4 \text { atm (1-2 } \mu \mathrm{s} \text { exposure). }
$$

$\Delta U \delta_{\omega} / \nu=2000$ reported by Breidenthal (1979) for the spanwise instability at $r=0.38$ but density ratio unity. Since the visual thickness is approximately twice the vorticity thickness (Konrad 1976), the critical Reynolds number based on the visual thickness is $\mathbf{4 0 0 0}$ for Breidenthal's result. This is the value found in figure 7 for the corresponding value of $\Delta U / U_{\mathrm{c}}=0.9$.

Several characterstic lengths are available for characterizing the initial spacing of the streak pattern. An appropriate reference length might be the initial thickness of the shear layer after it leaves the splitter plate. Apart from the difficulties of measuring this small thickness, there are well-known problems of defining the position of the initial thickness, compounded here by the fact that the shear layer is generated from boundary layers in two different gases. Therefore, we use for the reference length the wavelength of the initial Kelvin-Helmholtz instability wave $\lambda_{0}$, which can be easily measured on the photographs. In fact, this instability wave educes the appropriate length parameter and provides a convenient means of measuring it. Alternatively the spacing between primary vortices $\lambda_{i}$ at the beginning of the streak pattern could also be used as a reference length. The value of $\lambda_{i}$ is in general greater than $\lambda_{0}$ because amalgamations occur before the onset of the visible pattern. Both $\lambda_{0}$ and $\lambda_{i}$ were measured on instantaneous pictures like those shown in figures 3 and 5 . At each flow condition the values used are average values from two different realizations of the flow. The values of $\delta_{\mathrm{vi}}$ normalized by $\lambda_{0}$ are given in table 1. 


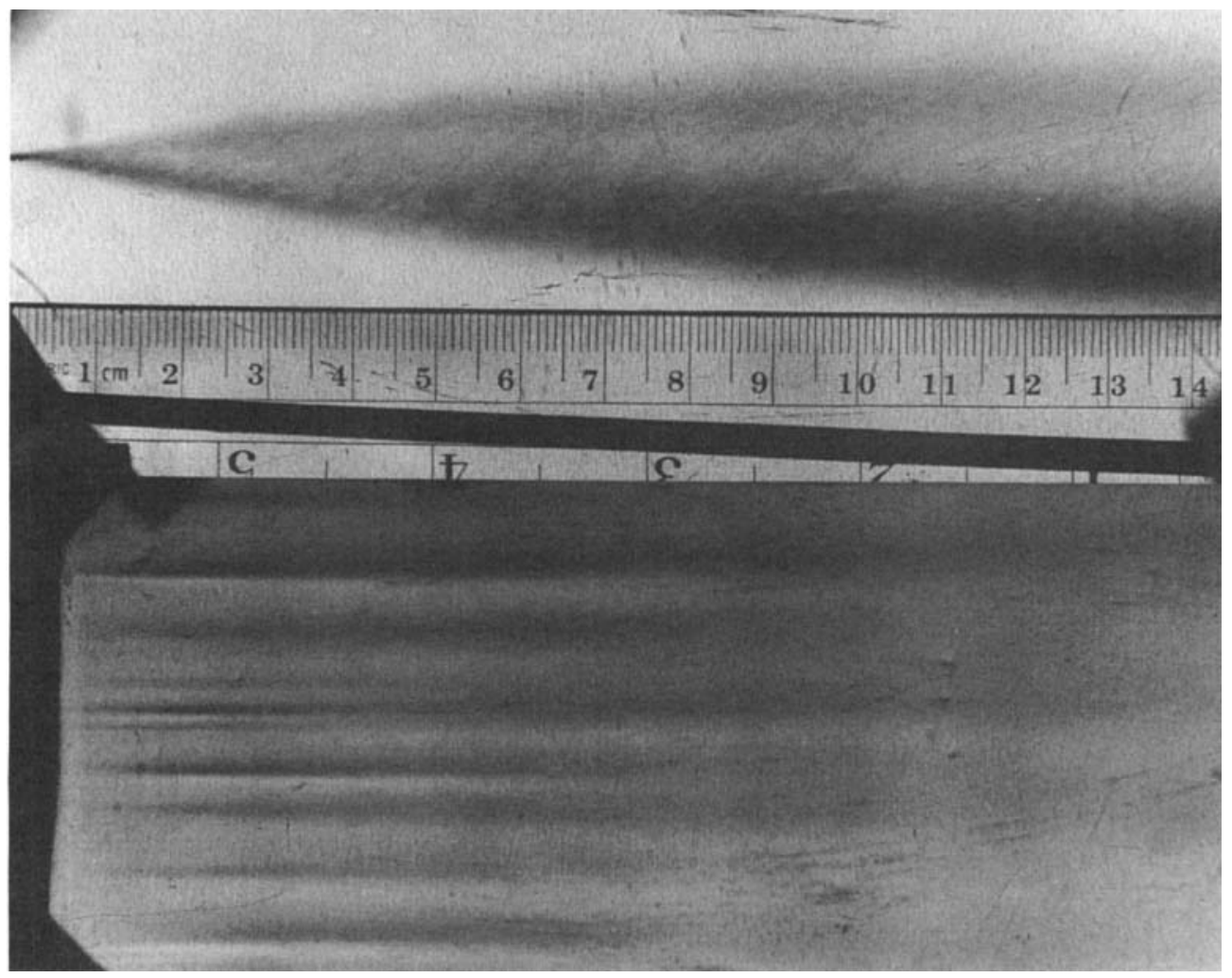

Figure 6. Time-exposure Schlieren picture of the helium-nitrogen mixing layer.

Same flow conditions as in figure 5 ( $2 \mathrm{~s}$ exposure).

\begin{tabular}{|c|c|c|c|c|c|c|c|c|c|}
\hline$\underset{\text { (atm) }}{P_{0}}$ & $\begin{array}{c}L_{\mathrm{LB}} \\
(\mathrm{cm})\end{array}$ & $r$ & $\Delta U / U_{\mathrm{c}}$ & $\begin{array}{c}U_{1} \\
(\mathrm{~cm} / \mathrm{s})\end{array}$ & $\begin{array}{c}\Delta U \delta_{\mathrm{vi}} / \nu_{2} \\
\left(\times 10^{-3}\right)\end{array}$ & $\delta_{\mathrm{v} 1} / \lambda_{0}$ & $s / \lambda_{0}$ & $s / \lambda_{i}$ & $s^{\prime} / \lambda_{t}$ \\
\hline 4 & 3.0 & 0.15 & 2.16 & $\begin{array}{r}1467 \\
992\end{array}$ & $\begin{array}{l}13.3 \\
14.8\end{array}$ & $\begin{array}{l}1.02 \\
1.11\end{array}$ & $\begin{array}{l}1.63 \\
1.25\end{array}$ & $\begin{array}{l}0.93 \\
0.68\end{array}$ & $\begin{array}{l}0.20 \\
0.16\end{array}$ \\
\hline - & - & 0.3 & 1.39 & $\begin{array}{r}1470 \\
998 \\
752\end{array}$ & $\begin{array}{r}10.1 \\
10.7 \\
9.6\end{array}$ & $\begin{array}{l}0.94 \\
1.16 \\
1.17\end{array}$ & $\begin{array}{l}1.31 \\
1.21 \\
1.22\end{array}$ & $\begin{array}{l}0.70 \\
0.67 \\
0.56\end{array}$ & $\begin{array}{l}0.28 \\
0.16 \\
0.08\end{array}$ \\
\hline - & - & 0.38 & 1.10 & $\begin{array}{r}1008 \\
740 \\
504\end{array}$ & $\begin{array}{l}6.1 \\
6.3 \\
6.7\end{array}$ & $\begin{array}{l}0.92 \\
1.30 \\
1.35\end{array}$ & $\begin{array}{l}1.29 \\
1.29 \\
1.20\end{array}$ & $\begin{array}{l}0.68 \\
0.51 \\
0.65\end{array}$ & $\begin{array}{l}0.27 \\
0.21 \\
0.05\end{array}$ \\
\hline - & - & 0.50 & 0.77 & $\begin{array}{r}1012 \\
755 \\
496\end{array}$ & $\begin{array}{l}3.3 \\
3.3 \\
3.1\end{array}$ & $\begin{array}{l}0.61 \\
0.83 \\
0.96\end{array}$ & $\begin{array}{l}1.17 \\
1.50 \\
1.42\end{array}$ & $\begin{array}{l}0.78 \\
0.75 \\
0.64\end{array}$ & $\begin{array}{l}0.30 \\
0.27 \\
0.09\end{array}$ \\
\hline 6 & 3.0 & 0.38 & 1.10 & $\begin{array}{r}1000 \\
651\end{array}$ & $\begin{array}{l}5.7 \\
7.0\end{array}$ & $\begin{array}{l}0.78 \\
1.11\end{array}$ & $\begin{array}{l}1.40 \\
1.25\end{array}$ & $\begin{array}{l}0.70 \\
0.56\end{array}$ & $\begin{array}{l}0.26 \\
0.23\end{array}$ \\
\hline 4 & 5.5 & 0.38 & 1.10 & $\begin{array}{l}996 \\
758\end{array}$ & $\begin{array}{l}9.5 \\
9.4\end{array}$ & $\begin{array}{l}1.05 \\
1.26\end{array}$ & $\begin{array}{l}1.47 \\
1.20\end{array}$ & $\begin{array}{l}0.67 \\
0.66\end{array}$ & $\begin{array}{l}0.17 \\
0.12\end{array}$ \\
\hline
\end{tabular}

TABLE 1. Initial streak-pattern measurements, $\rho_{2} / \rho_{1}=7$. 


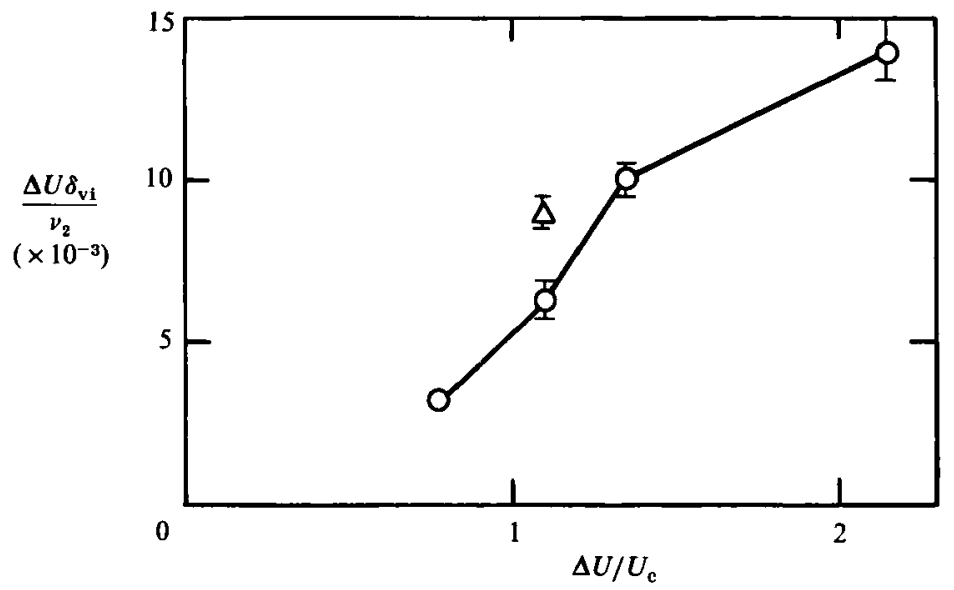

Figure 7. Critical Reynolds number of streamwise streak-pattern dependence on $\Delta U / U_{\mathrm{c}}, \rho_{2} / \rho_{1}=7 . \bigcirc$, standard partition; $\Delta$, extended partition.

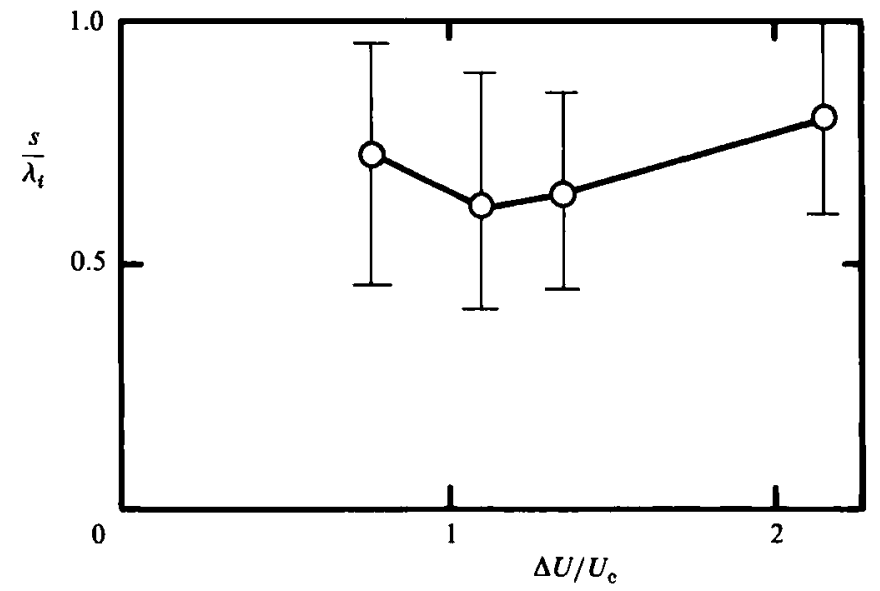

FigURE 8. Streamwise streak-spacing dependence on $\Delta U / U_{\mathbf{c}}, \rho_{2} / \rho_{1}=7$.

The initial spanwise spacing of the streaks was measured on the time-exposure pictures. The mean spanwise spacing $s$ normalized by both $\lambda_{0}$ and $\lambda_{i}$ are given in Table 1. The values of $s / \lambda_{0}$ can be compared with the result of Breidenthal for the spanwise wavelength of the instability $s / \lambda_{0}=1.1$. The results of the present measurements at $\rho_{2} / \rho_{1}=7$ are typically $20 \%$ larger than the value reported by Breidenthal at uniform density. The standard deviation of the spacing normalized by $\lambda_{i}$ at each flow condition is given in the last column of table 1 . Its value increases with flow velocity. It can be seen that the variation of mean spacing between different cases is smaller than the standard deviation. This is clearly seen in figure 8 where $s / \lambda_{i}$ is plotted as a function of $\Delta U / U_{c}$. The bars on this figure indicate the standard deviation computed from all samples at a given value of $\Delta U / U_{\mathrm{c}}$. We conclude from these results that the spanwise spacing normalized by $\lambda_{i}$ is independent of the velocity ratio and initial shear-layer profile. The mean value of $s / \lambda_{i}$ calculated from all samples available is 0.67 . Similarly the normalized standard deviation $s^{\prime} / \lambda_{i}=0.43$ using all samples. The most significant contribution to this deviation is the large scatter found in individual realizations; any effects of changing flow parameters are masked by it. 


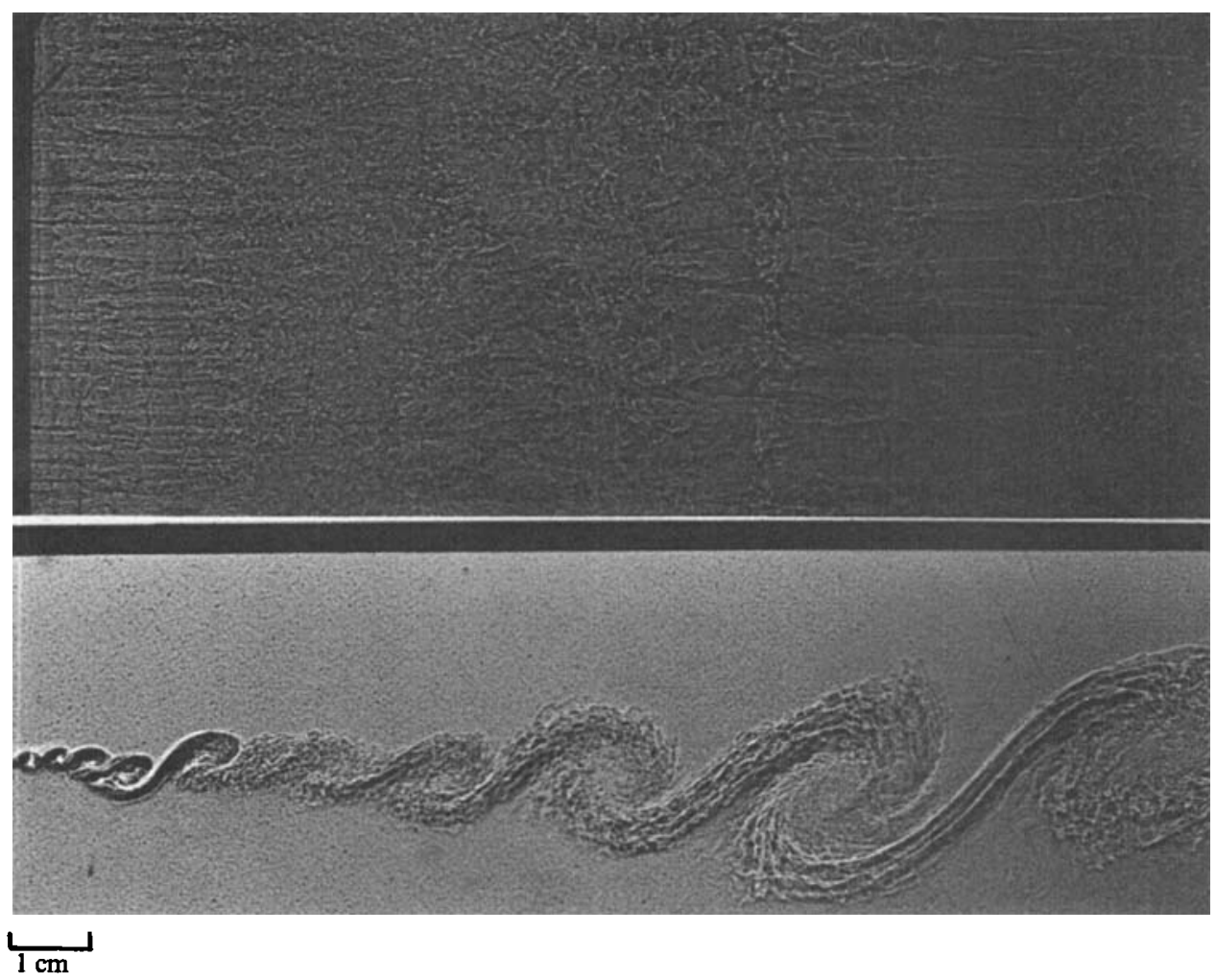

Figure 9. Spark shadowgraph picture of the uniform-density mixing layer. $U_{1}=1000 \mathrm{~cm} / \mathrm{s}$, $r=0.38, P_{0}=4 \mathrm{~atm}$. Scale as indicated. From Konrad (1976).

The time-exposure pictures show loss of definition of the streamwise streak pattern at sufficient distance downstream, that is at sufficiently high Reynolds number, as in figure 6. The gradual loss of contrast is accompanied by spacing changes of the streak pattern with downstream distance. Additional qualitative evidence on the downstream evolution of the streaks was obtained from high-speed shadowgraph motion pictures, which showed that the streaks originate at fixed spanwise positions. Further downstream changes in the pattern develop. The lateral positions of the streaks at a given downstream location fluctuate in time and the number of streaks across the span decreases with increased distance downstream. This behaviour is consistent with the time-exposure pictures since fluctuation of lateral position and spacing will result in loss of the initial coherence of the pattern. In all flow conditions considered in this investigation the streamwise streaks were observed in instantaneous pictures even at the highest Reynolds numbers and at the downstream end of the test section far downstream of the mixing transition.

An example of the development of the streaks, obtained in the same apparatus by Konrad (1976), is reproduced in figure 9. In Konrad's experiments, the gas on the low-speed side (a mixture of helium and argon) had the same density as the high-speed, nitrogen stream, i.e. $\rho_{2} / \rho_{1}=1$. The conditions are the same as for our helium-nitrogen mixing layer at $U_{2} / U_{1}=0.38$ and $P_{0}=4 \mathrm{~atm}$ (see table 1), except that $\rho_{2} / \rho_{1}=1$ rather than 7 . The trailing edge of the partition is to the left of the edge of the photograph. At the edge $\Delta U \delta_{v} / \nu \approx 5000$ (see Brown \& Roshko 1974, for the method of estimating $\delta_{\mathrm{v}}$ ). From the data in table 1 and Breidenthal's (1979) 

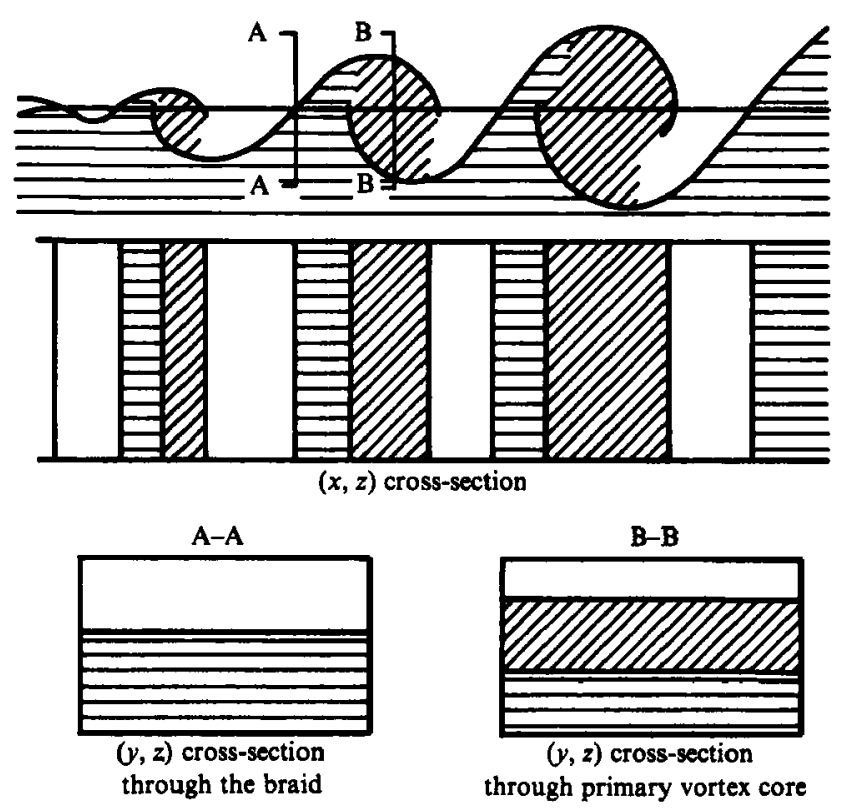

Figure 10. Cross-section-views schematic.

, labelled low-speed fluid. $\mathbf{D}$, mixed fluid.

results this would be the origin of the streamwise streaks on long time exposures (not obtained by Konrad). A relevant feature of this photograph is the marked appearance of small-scale turbulent structure about $2 \mathrm{~cm}$ downstream of the left edge of the picture. We interpret this to be the instantaneous location of the 'mixing transition' which was first observed by Konrad. At this location $\Delta U \delta_{\omega} / \nu \approx 6500$ and $x / \theta \approx 280$, where $\theta$ is the momentum thickness of the boundary layer on the high-speed side of the partition, which agree well with the end of the mixing transition measured by Breidenthal (1981). Thus, in this example, $\delta_{\omega}$ increases by a factor of 2.5 from initiation of the streaks to completion of mixing transition. Downstream of the mixing transition the streaks are clearly visible to the right of the photograph. The spacing has increased compared with the initial value. This aspect will be further discussed below.

\section{Streamwise vortex topology}

In this section we discuss the topology of the flow structure associated with the streamwise streaks and the relation of this structure to the spanwise coherent vortices. Laser-induced fluorescence was used to visualize cross-sections of the flow as shown schematically in figure 10 . In the $(x, z)$ cross-section, fluid from both streams meet along a thin interface, the 'braid', idealized as a thin line in the figure. Fluid from the low-speed stream is at the downstream side of the braid while fluid from the high-speed stream is at the upstream side. Mixed fluid in the spanwise vortex core also separates unmixed fluids. The spanwise core is preceded on the downstream side by fluid from the high-speed stream and followed by fluid from the low-speed stream. The $(y, z)$ cross-sections vary depending on the relative position of the primary vortex. For example cross-section A-A in figure 10 is a slice through the braid while cross-section B-B is a slice through a spanwise vortex core. As we shall see below, 


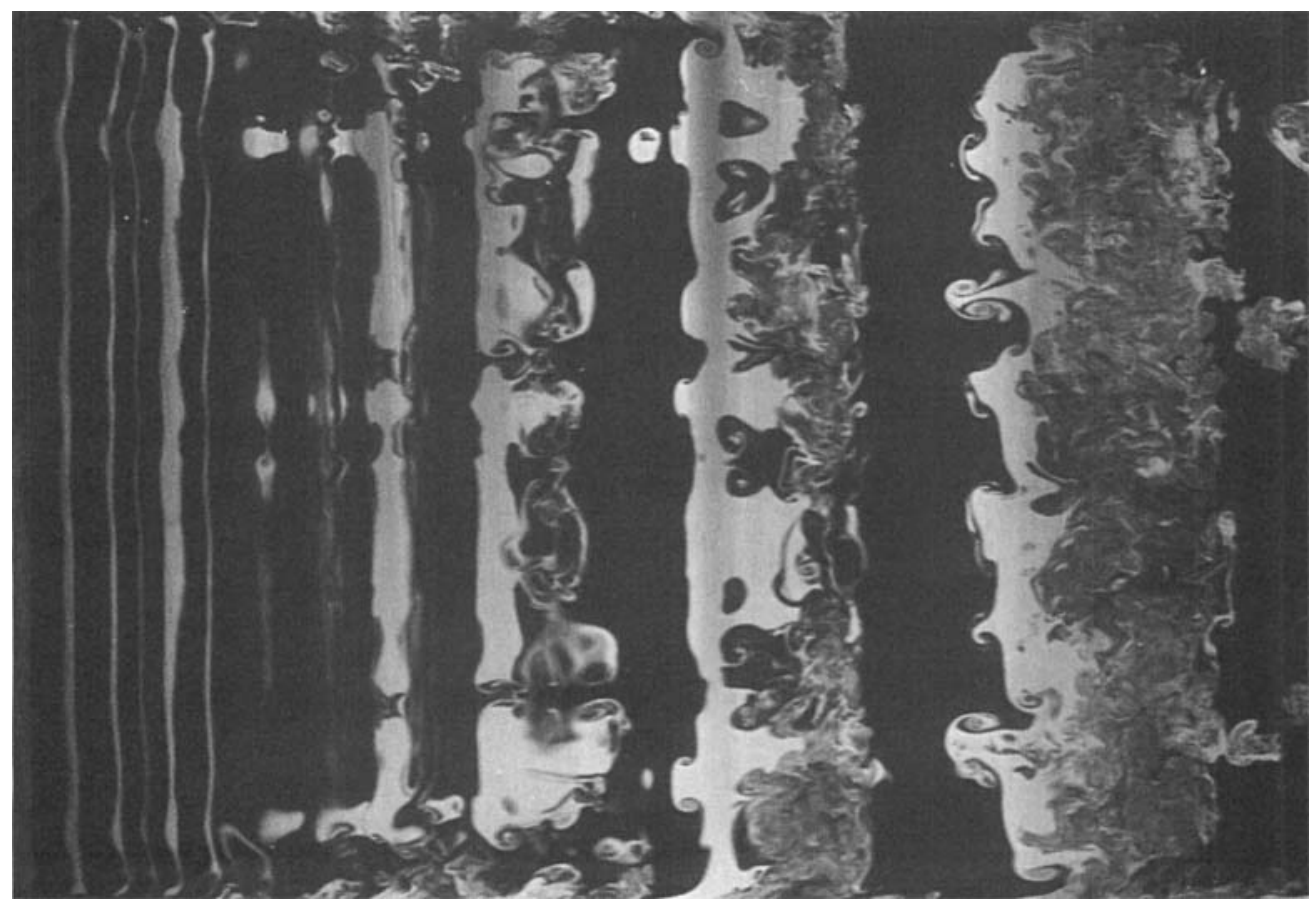

Figure 11. Laser-induced fluorescence $(x, z)$-cross-section picture. $U_{1}=57 \mathrm{~cm} / \mathrm{s}, U_{2}=23 \mathrm{~cm} / \mathrm{s}$. Flow is from left to right. The entire channel span $(11 \mathrm{~cm})$ is shown.

the streamwise vortices are located on the braids and on the periphery of the spanwise vortex cores.

An $(x, z)$ cross-section of a mixing layer in water is presented in figure 11 . The flow is from left to right. The velocity ratio $r$ was 0.4 and the Reynolds number per unit length was $2300 \mathrm{~cm}^{-1}$. This picture shows the early development of threedimensionality in the mixing layer, from the two-dimensional roll-up of the KelvinHelmholtz instability wave at the left of the picture, to the appearance of streamwise counter-rotating vortices on the braid between the last two spanwise vortices. Although passive dye visualization does not visualize vorticity directly, the presence of counter-rotating vortices is inferred from the swirled interface. The threedimensional instability is developing on the spanwise vortex at the centre of the photograph. From measurements on this picture we estimate a local Reynolds number $\Delta U \delta_{\omega} / \nu=1900$ and $s / \lambda_{i}=0.6$ where $\lambda_{i}$ is an average value based on the two spanwise vortices at the centre of the photograph. Both estimates are in good agreement with the results presented in the previous section.

Figures 12 and 13 are $(y, z)$ cross-section photographs of the mixing layer obtained at a Reynolds number based on the local vorticity thickness $\Delta U \delta_{\omega} / \nu=3000$. The visualized plane is $15 \mathrm{~cm}$ from the splitter plate $(x / \theta \approx 200)$. The flow is perpendicular to the plane of the picture and towards the viewer. The high-speed stream is on top, the low-speed stream, carrying the labelled fluid, is at the bottom. Figure 12 is a slice through the braid while figure 13 is a slice through the spanwise vortex core. On the braid there is a single row of counter-rotating vortices. Their footprint on this figure and on figure 11 suggests that the vortex lines are aligned with the braids. The slice 


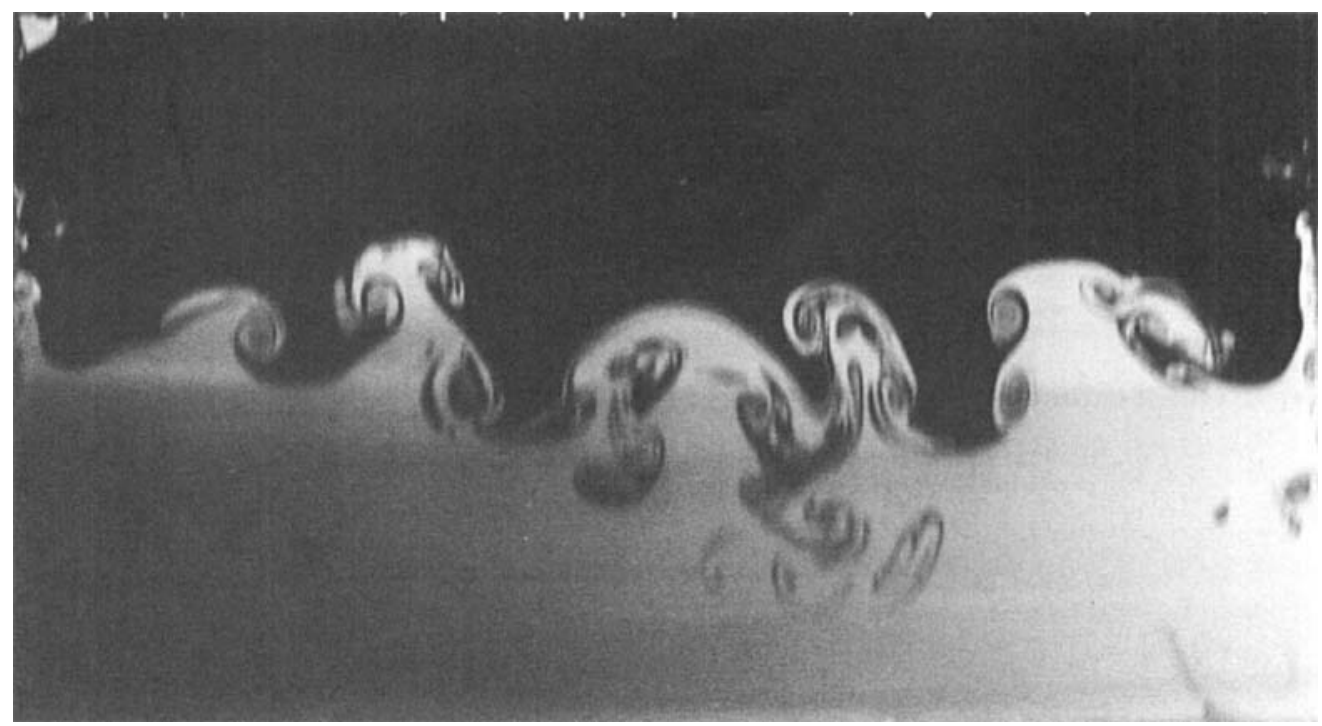

FIGURE 12. Laser-induced fluorescence $(y, z)$ cross-section through the braid. $U_{1}=29 \mathrm{~cm} / \mathrm{s}, U_{2}=10 \mathrm{~cm} / \mathrm{s}$. The entire channel $\operatorname{span}(11 \mathrm{~cm})$ is shown.

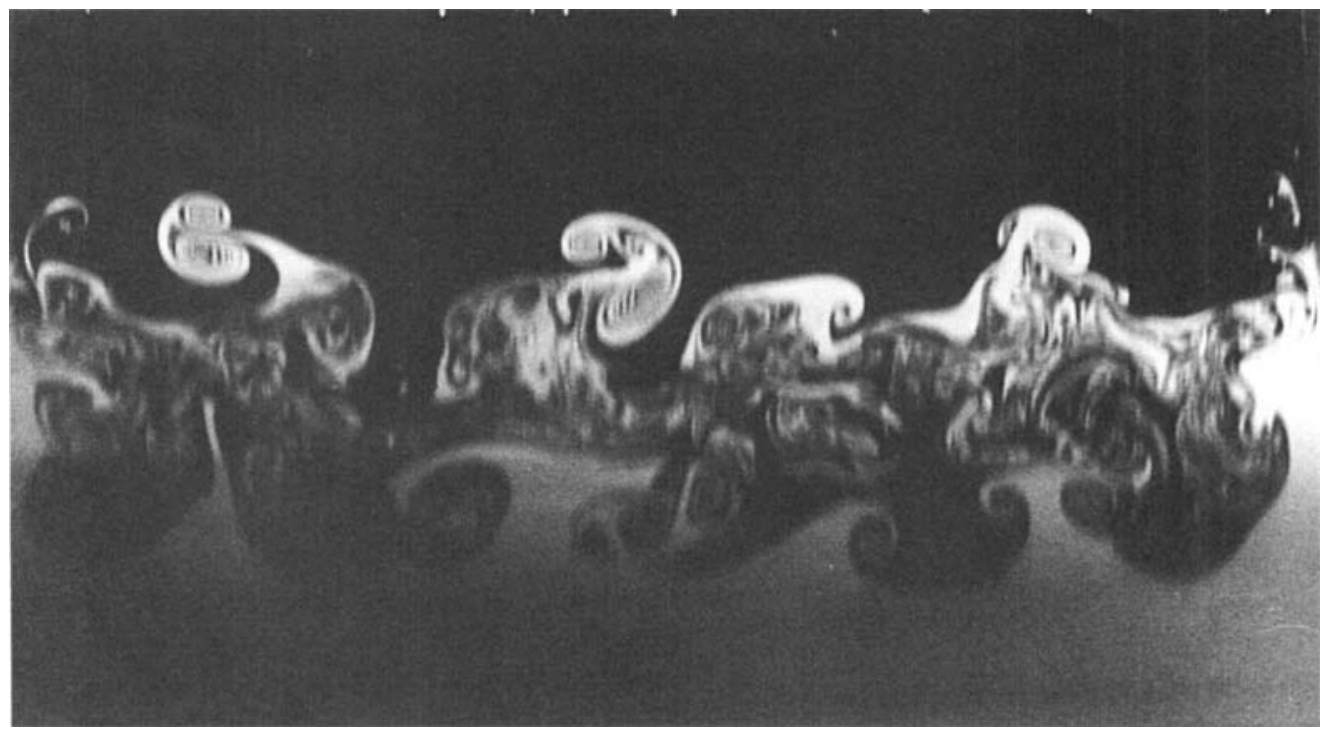

Figure 13. Laser-induced fluorescence $(y, z)$ cross-section through the core. $U_{1}=20 \mathrm{~cm} / \mathrm{s}, U_{2}=8 \mathrm{~cm} / \mathrm{s}$. The entire channel span $(11 \mathrm{~cm})$ is shown.

through the spanwise vortex core, figure 13, shows two rows of counter-rotating vortices located on the top and bottom of the core, respectively.

The topology of the streamwise-vortex structure and its relation to the spanwise vortices was further investigated with high-speed motion pictures obtained at a Reynolds number $\Delta U \delta_{\omega} / \nu=2400$. The visualized plane is $15 \mathrm{~cm}$ from the splitter plate $(x / \theta \approx 330)$. A sequence from these pictures is shown in figure 14 . It may be helpful in following the foregoing discussion to refer to figure 15, whose construction was based on this and other sequences. The sequence in figure 14 shows the passage 


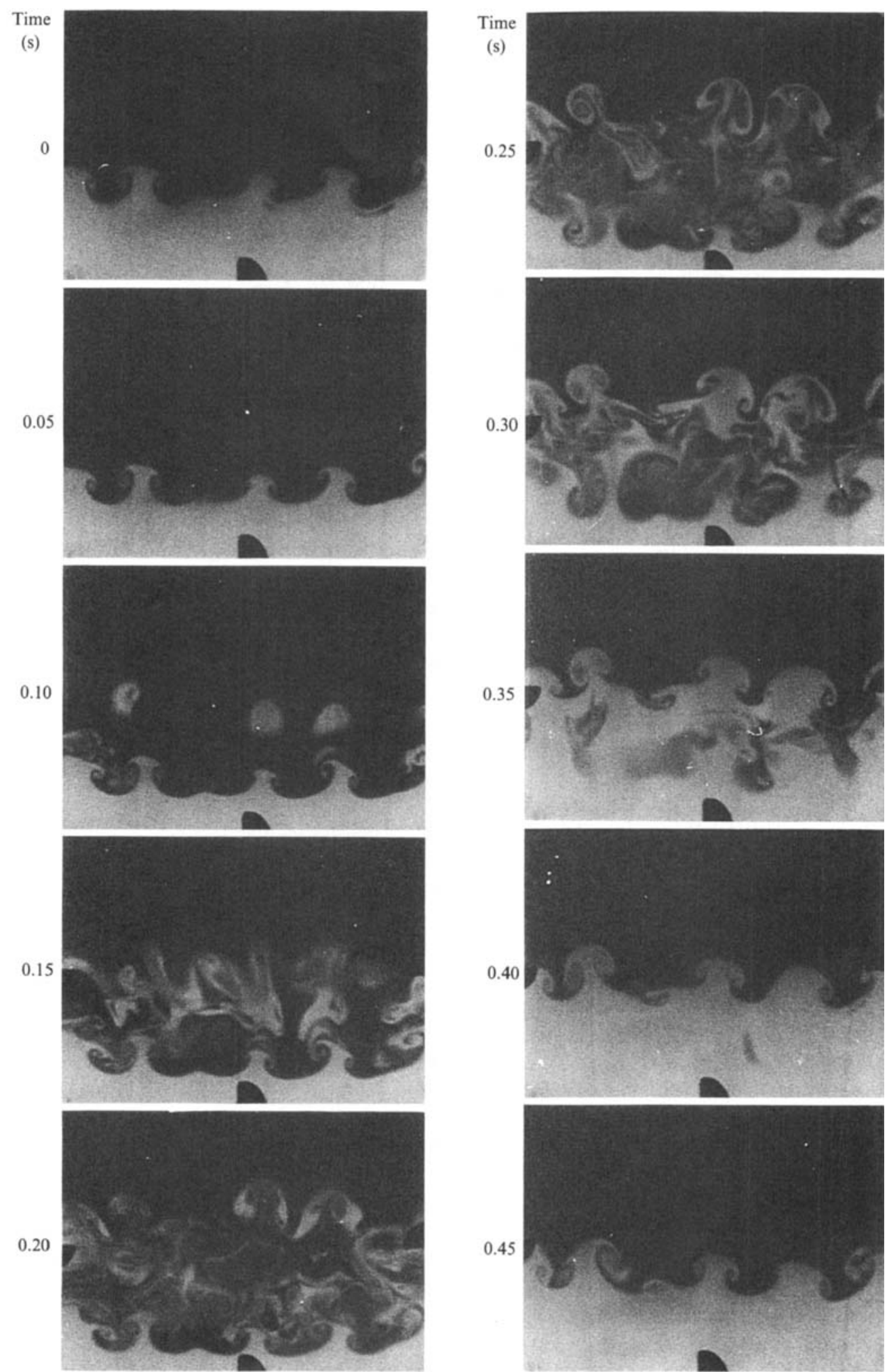

Fiqure 14. Laser-induced fluorescence $(y, z)$-cross-section film sequence. $\Delta U \delta_{\omega} / \nu=2.4 \times 10^{3}$. 


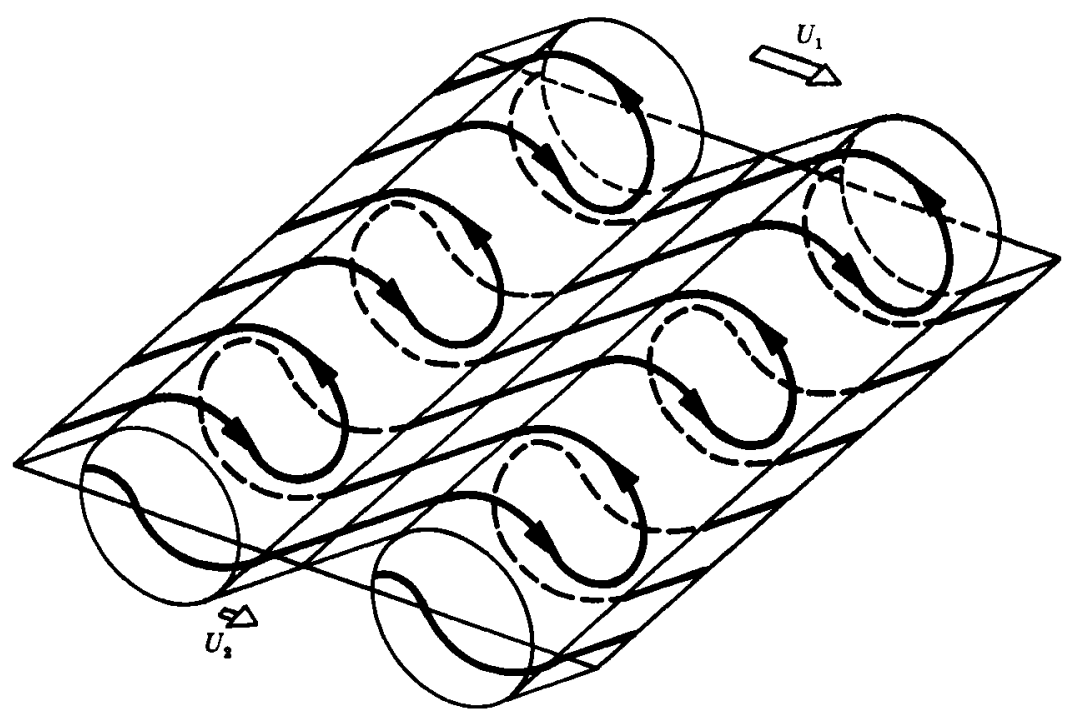

Figure 15. Topology of streamwise vortex lines.

through the illuminated plane of a spanwise vortex. This particular sequence was chosen because the spanwise vortex is not influenced by an amalgamation process as it moves through the cross-section. In the first two pictures of the sequence the braid is seen to move towards the low-speed side, and the streamwise vortices follow this motion. First contact of the primary vortex core with the visualized plane is seen in the third frame $(t=0.1 \mathrm{~s})$. It occurs at isolated regions across the span indicating outflow of fluid from the core in these areas; furthermore, the location of these outflow regions correlate well with the location of the counter-rotating vortices, in particular with the positions where their induced velocities are directed upwards. As the core moves through the visualized plane, a second row of counter-rotating vortices appears on the high-speed side of the spanwise vortex core. Vortex pairs on the high-and low-speed sides of the primary vortex are aligned with each other in such a way as to have the same sign vorticity at the same spanwise location. The subsequent evolution shows the streamwise vortices at the low-speed side disappearing and the primary vortex core leaving behind a region of outward flow from the core $(t=0.35 \mathrm{~s})$, i.e. normal to the picture plane and upstream. The streamwise vortices at the high-speed side continue along the trailing braid, following it as it moves toward the low-speed side. The outflow regions observed prior to the appearance of the streamwise vortices on the high-speed side and after the disappearance of those at the low-speed side indicates that the streamwise vortex lines bend around the periphery of the large-scale vortex core.

No evidence was found of the continuation of the streamwise vortices along the periphery of the primary vortex core beyond the regions where the vortex lines are perpendicular to the plane of the mixing layer, which suggests that the vortices in each counter-rotating pair join in a hairpin configuration. Although direct evidence of the presence of the hairpins was not obtained, this conjecture is consistent with the observations by Breidenthal (1981) of the early development of threedimensionality; he proposed that stretching of a spanwise deformation of the 
primary vortex (the 'wiggle') leads to the formation of the streamwise streaks. This possibility has also been suggested by Jimenez (1983).

Another feature of the interaction between streamwise and primary vortices is the tendency of the streamwise vortices to move away from the core. This trend can be observed in the movie sequence $(t=0.2,0.25)$ as well as in figure 13 . It is associated with curvature of the streamwise-vortex lines imposed by the primary-vortex velocity field. The curvature induces a precessional motion which causes uneven spacing between the streamwise vortices on the periphery of the primary vortex. The resulting counter-rotating pairs have self-induced radial velocities directed outwards, away from the primary vortex core.

Based on these pictures, the idealized diagram of the vortex lines presented in figure 15 was constructed. A streamwise vortex segment is part of a continuous line which loops back and forth between adjacent primary vortices as shown in the figure. Thus on successive passes, its streamwise direction changes sign, forming the vortex pairs that are seen in the cross-section pictures. These warped vortex structures, linking adjacent pairs of primary vortices, align themselves with each other so that, in plan view they appear to be straight streamwise streaks (e.g. figures 3 and 9). Actually, in the ideal picture the projections onto a plan view would not really be straight but would have deflections due to the induced precessional motions described above. On shadow and Schlieren pictures, these details, superimposed on other features from the internal structure of the primary vortex, are obscured. A slightly different view of the streamwise-vortex structure has been proposed by Hussain (1983). His model was derived from phase-averaged turbulent production measurements. It must be emphasized that figure 15 is an idealized model which incorporates the essential features of the secondary structure. Instantaneous realizations like those in figures 11-14 reveal a number of interesting variations and intriguing interactions within this structure. These were not explored further in this investigation. They are however consistent with the proposed model.

\section{Spanwise concentration non-uniformities}

The streamwise streak pattern in the time-exposure photographs made in the gas flows is caused by concentration gradients in the mean flow. The cross-section pictures in the water flows show significant spanwise concentration non-uniformities associated with the streamwise vortex structure. Concentration measurements were undertaken in the gas facility to obtain a quantitative description of these nonuniformities and to provide additional insight into the underlying streamwise vortex structure. Also we wished to see whether any mean spanwise structure actually remains in regions downstream of where it has disappeared from the time-exposure photographs. The measurements were limited to a single flow condition, namely $U_{1}=1000 \mathrm{~cm} / \mathrm{s}$ for the helium stream, $U_{2}=380 \mathrm{~cm} / \mathrm{s}$ for the nitrogen stream and test-section pressure at 6 atm. The Reynolds number per unit length was $\Delta U / \nu_{2}=24000 \mathrm{~cm}^{-1}$. At these flow conditions, $r=0.38$ and $\rho_{2} / \rho_{1}=7$, the mixing transition is complete at a Reynolds number, based on vorticity thickness, of 20000 according to the measurements of Konrad (1976). He also reported a vorticity thickness growth rate $\mathrm{d} \delta_{\omega} / \mathrm{d} x=0.12$ for these flow conditions.

Mean and r.m.s. concentration profiles were measured at distances from the splitter plate of $x=3$ to $11 \mathrm{~cm}$ on the midspan plane $(z=0)$. We define the concentration thickness $\delta$ as the distance across the layer between points where the 


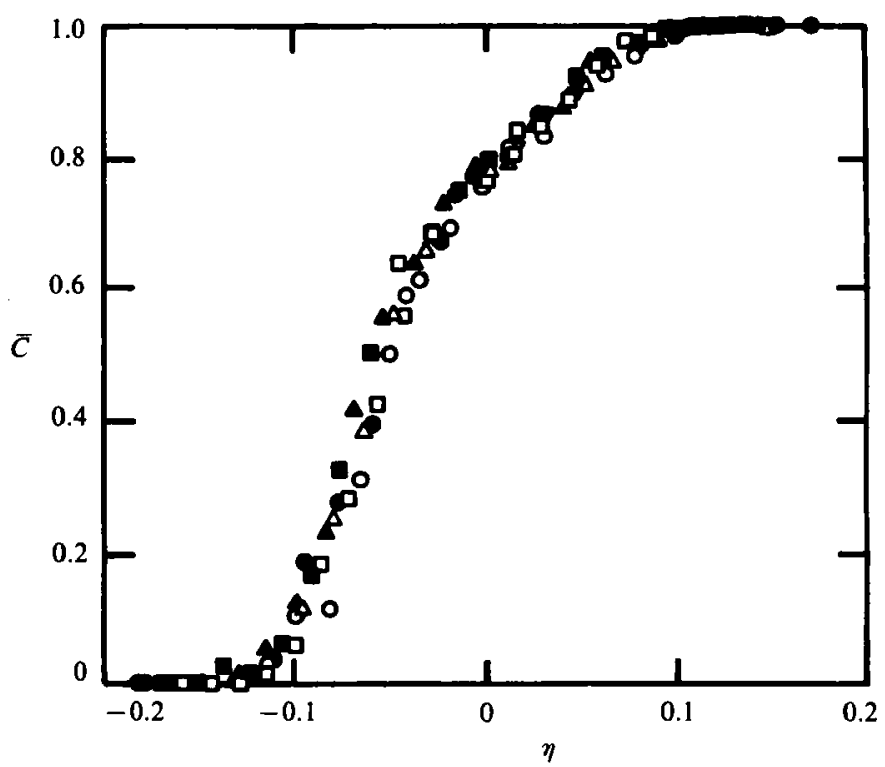

Figure 16. Mean concentration profiles.,$x=6 \mathrm{~cm} ; \triangle, 7 \mathrm{~cm} ; 0,8 \mathrm{~cm}$; $\Delta, 9 \mathrm{~cm} ; \square, 10 \mathrm{~cm} ; \square, 11 \mathrm{~cm}$.

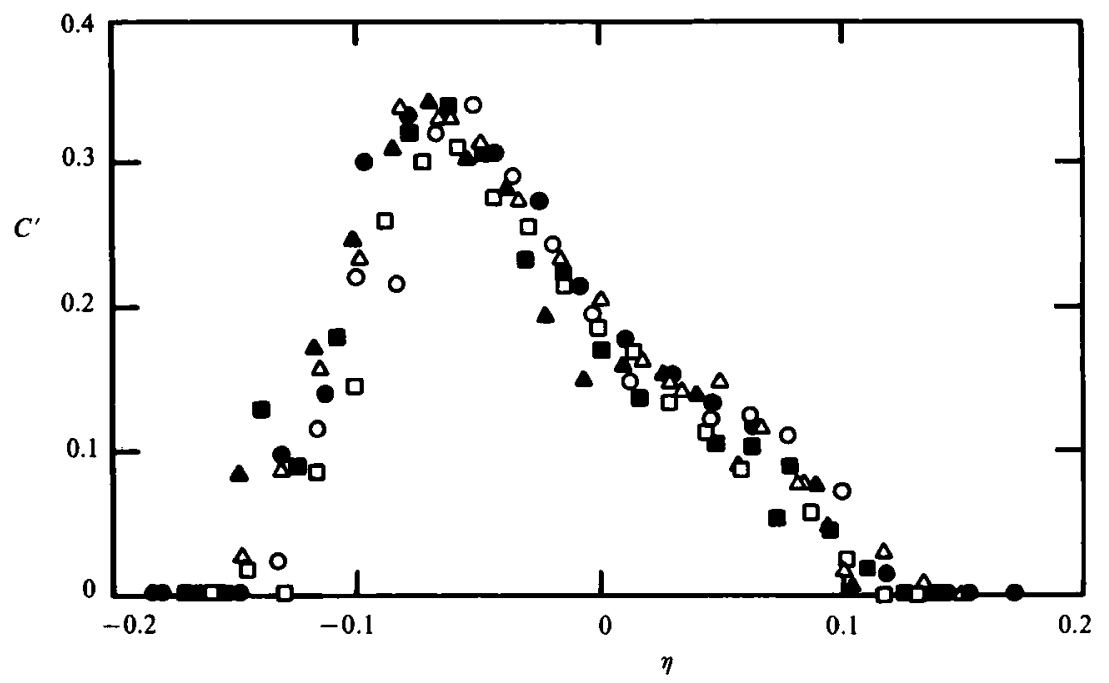

Figure 17. Profiles of the r.m.s. concentration fluctuation. For symbols see figure 16.

concentration deviates 0.01 from the free-stream value (Brown \& Roshko, 1974). The measured concentration thickness is given by

$$
\delta=0.22\left(x-x_{0}\right), \quad x_{0}=-1.58 \mathrm{~cm} .
$$

The standard deviation from this line was $0.08 \mathrm{~cm}$. The growth rate, 0.22 , and the location of the virtual origin, $x_{0}$, are in good agreement with the results of Brown $\&$ Roshko at a density ratio $\rho_{2} / \rho_{1}=7$. We use this value of $x_{0}$ to define the similarity variable $\eta=y /\left(c-x_{0}\right)$. The mean concentration profiles are plotted in figure 16 in terms of this similarity variable $\eta$. The r.m.s. values of the concentration fluctuation 


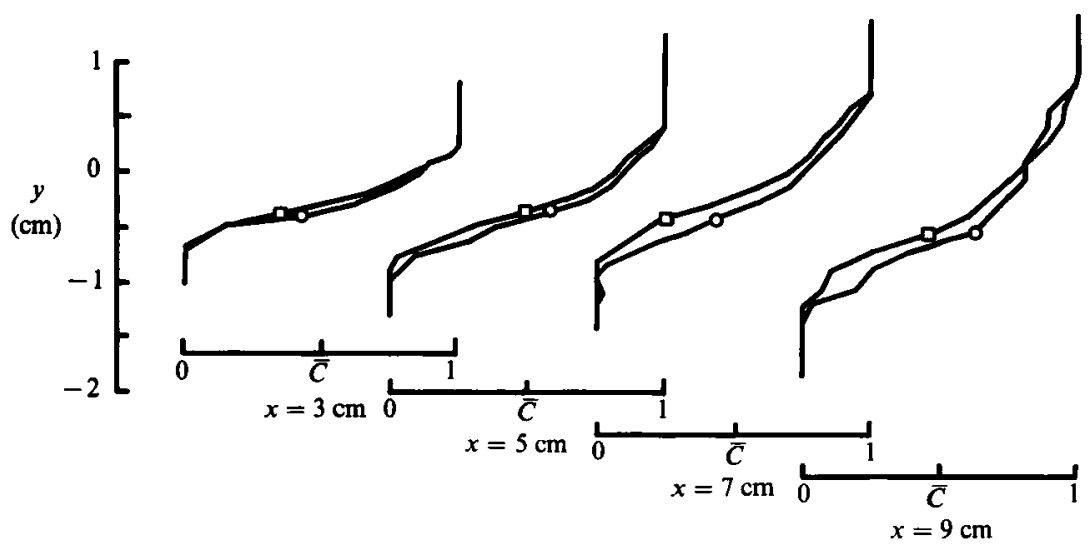

FIGURE 18. Streamwise evolution of mean-concentration profiles at two spanwise positions: $O, z=0 ; \square, 0.64 \mathrm{~cm}$. (Measured points in the profile are connected by straight-line segments.)

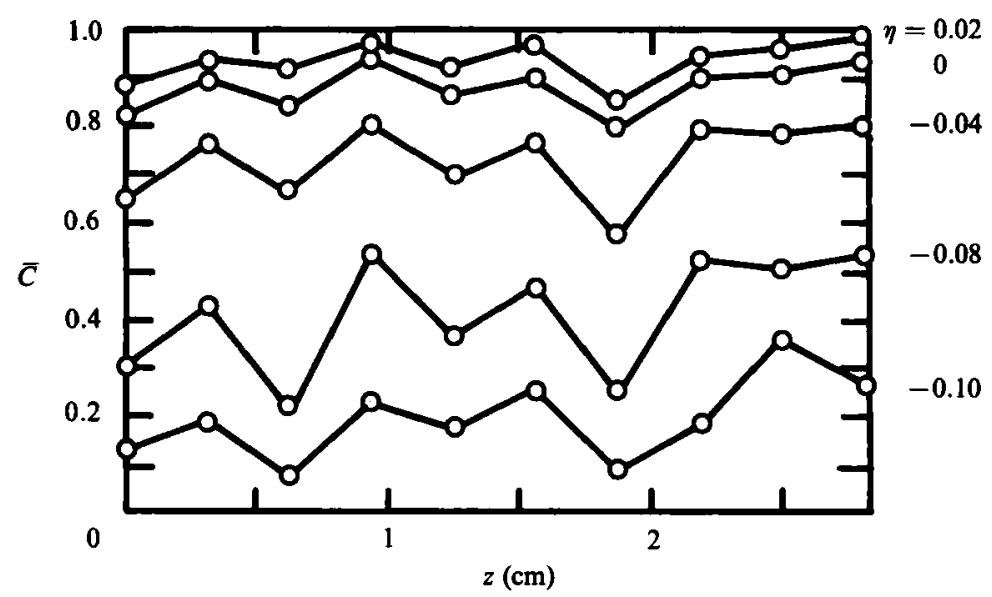

Fiaure 19. Spanwise variation of the mean concentration at $x=4 \mathrm{~cm}$.

are plotted in figure 17, also in terms of $\eta$. The profiles in both figures were measured at $x \geqslant 6 \mathrm{~cm}$, which is downstream of the mixing transition. These profiles, in the centre plane of the test section, are in good agreement with previous measurements by Brown \& Roshko (1974) and Konrad (1976).

Once the correspondence with earlier results had been established, we proceeded to measure spanwise variations with two concentration probes, one located at midspan and the other at several spanwise positions. The downstream evolution of the mean-concentration profiles differs depending on spanwise position. With the probes spaced $0.64 \mathrm{~cm}$ apart, pairs of profiles were obtained at four downstream locations as shown in figure 18. The probes were first placed on neighbouring streaks at $x=3 \mathrm{~cm}$ as indicated by time-exposure pictures, thus the close agreement at $x=3 \mathrm{~cm}$ is expected. As the probes were moved downstream, keeping the spacing constant, the profiles no longer overlap. As will be shown later, this is the result of an increased spacing between streaks.

To study this more completely, the mean concentration and the r.m.s. fluctuation were mapped over part of the $(y, z)$-cross-section plane, at two downstream locations, 


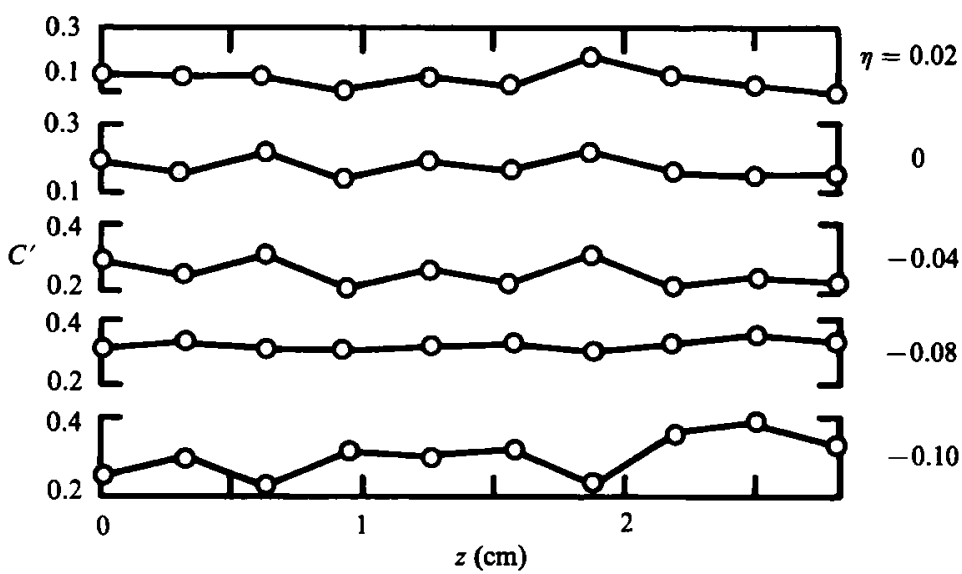

Fiaure 20. Spanwise variation of the r.m.s. value of the concentration fluctuation at $x=4 \mathrm{~cm}$.

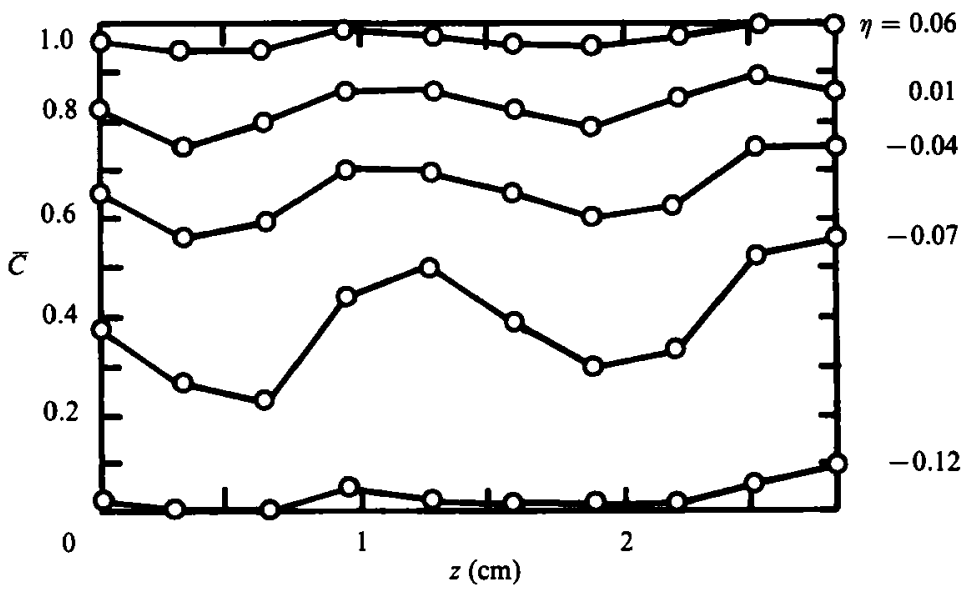

Fiaure 21. Spanwise variation of the mean concentration at $x=9 \mathrm{~cm}$.

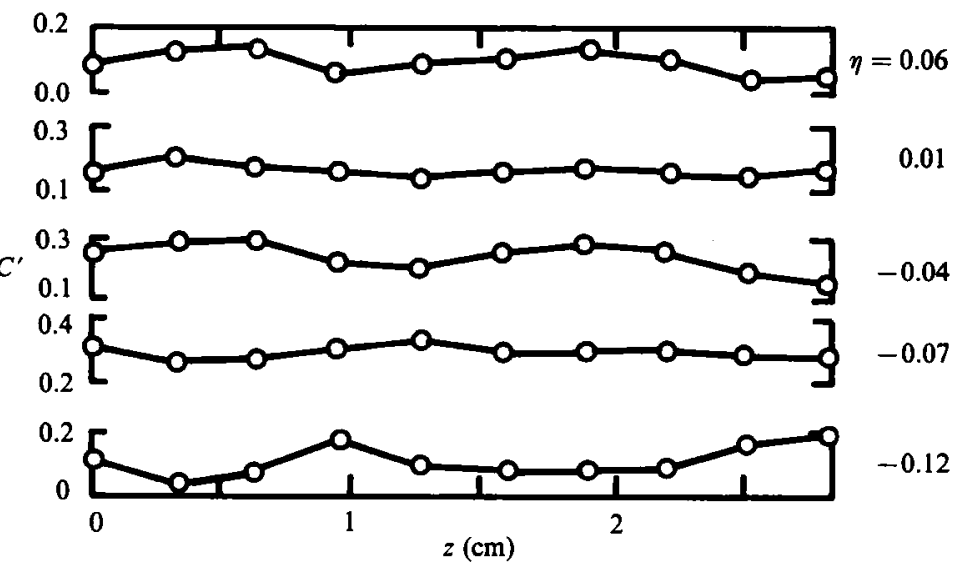

FIGURE 22. Spanwise variation of the r.m.s. value of the concentration fluctuation at $x=9 \mathrm{~cm}$. 


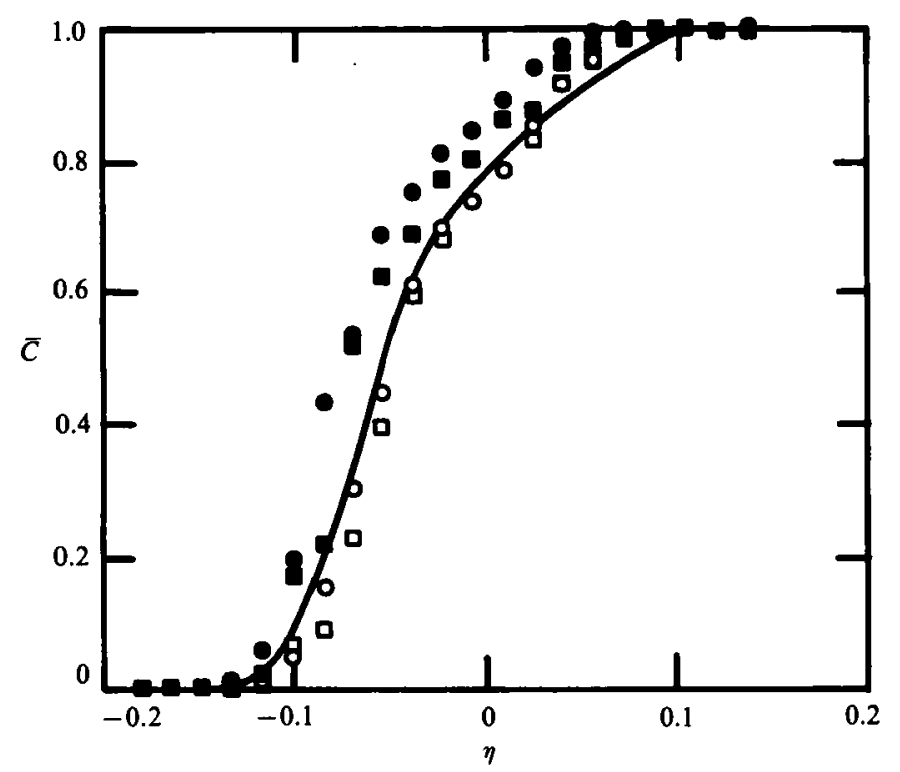

Figure 23. Mean concentration profiles measured at various spanwise positions: $\square, z=0.64 \mathrm{~cm} ; \square, 1.27 \mathrm{~cm} ; 0,1.91 \mathrm{~cm} ; 0,2.54 \mathrm{~cm} . \longrightarrow$, similarity profile from figure 16 .

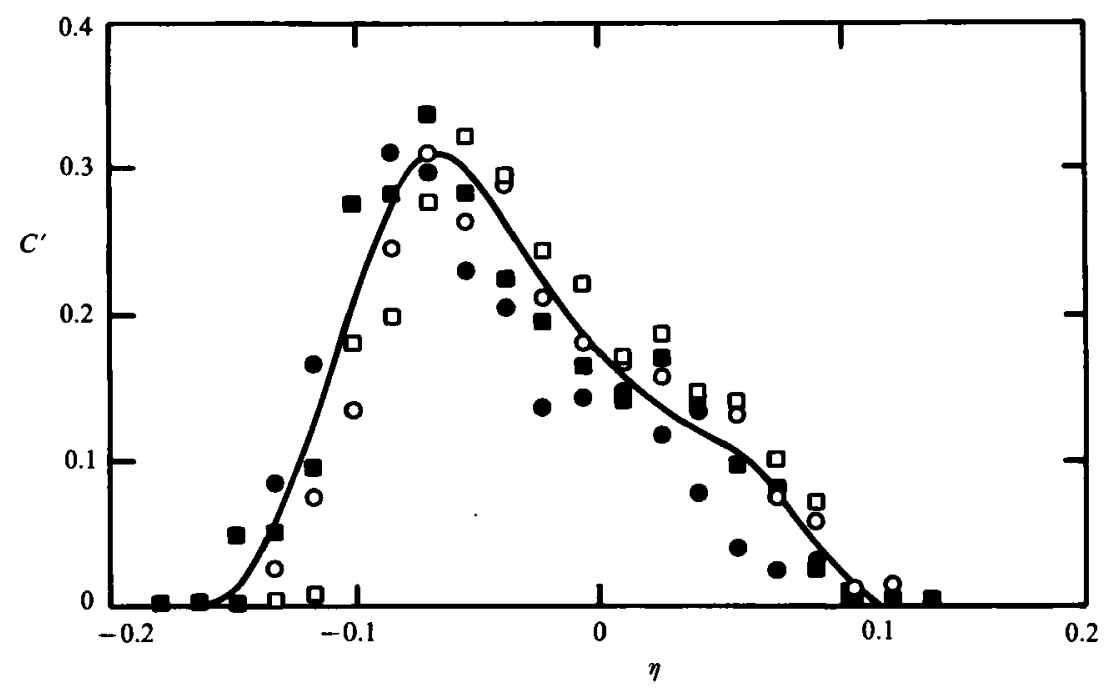

Figure 24. R.m.s. concentration fluctuation profiles at various spanwise positions. For symbols see figure 23 . , similarity profile from figure 17 .

$x=4 \mathrm{~cm}$ and $x=9 \mathrm{~cm}$. The local Reynolds number at $x=4 \mathrm{~cm}$ is $\Delta U \delta_{\omega} / \nu_{2}=16000$ while at $x=9 \mathrm{~cm}$ it is 30000 . Thus these stations are located upstream and downstream of the end of the mixing transition respectively. Figure 19 shows the spanwise variation of the mean concentration at several locations across the layer for $x=4 \mathrm{~cm}$. The peaks or valleys are at the same spanwise positions across the layer. The r.m.s. fluctuation measured at the same locations in the flow are presented in figure 20. They show similar oscillatory features. In this case, however, peaks in the nitrogen side $(\eta<-0.08)$ correspond to valleys on the helium side and peaks on the mean concentration. Further downstream at $x=9 \mathrm{~cm}$, figures 21 and 22 show the 

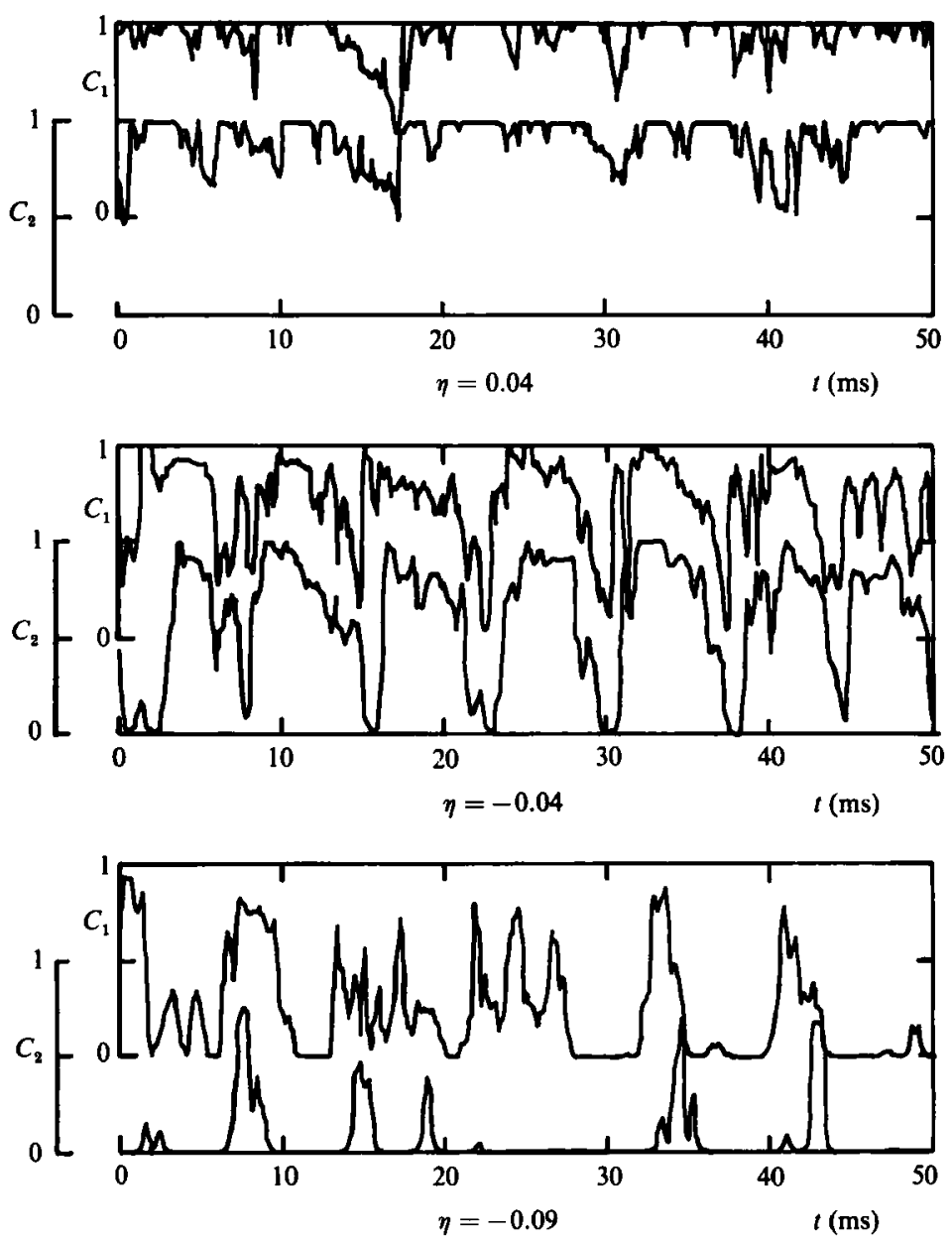

Figure 25. Comparison of concentration time histories at $z=0, C_{1}$, and $z=0.64 \mathrm{~cm}, C_{2}$, at $x=9 \mathrm{~cm}$ and three locations in the profile.

same features in the mean concentration and the r.m.s. fluctuation respectively. Using the spanwise spacing defined by the central part of figure 21 gives $s=1.25 \mathrm{~cm}$. Therefore only eight waves cover the span of the facility. This may have contributed to their fixed spanwise location in spite of the randomization mechanisms observed in the flow-visualization pictures. The spanwise spacing between peaks and valleys has increased compared with the value of $s=0.6 \mathrm{~cm}$ at the upstream location. In terms of the local vorticity thickness we find $s / \delta_{\omega}=1.0$ at $x=9 \mathrm{~cm}$. Nearly the same dimensionless value for $s$ is calculated from the three wavelengths available in figure 19. Between $x=4 \mathrm{~cm}$ and $x=9 \mathrm{~cm}$ the change in basic scale is by a factor of 1.9 (in $\delta_{\omega}$ ) and, evidently, the same is true for the spanwise structure. The amplitude of the mean concentration spanwise oscillations is the same at the two downstream positions (figures 19 and 21 ). The maximum r.m.s. value of this spanwise variation is 0.10 at $\eta \approx 0.07$ (which is the location of the maximum amplitude of the time fluctuation in figure 17 ). The value of the mean concentration there is $\bar{C} \approx 0.35$.

The spanwise changes of mean properties occur across the entire layer. Mean and r.m.s. concentration profiles measured at $x=9 \mathrm{~cm}$, at several locations along the 
span, are presented in figures 23 and 24 respectively. In both figures solid symbols are used for profiles measured at a peak and open symbols are used for those measured at a valley. Aslo plotted in these figures are lines fitted to the similarity profiles in figure 16 and 17 respectively. At the peaks the profiles are displaced towards the nitrogen side while at the valleys the displacement is towards the helium side. These displacements are associated with the velocity field induced by the secondary vortices. They indicate preferential entrainment of fluid from each stream at different spanwise locations. Consistent with this view, the r.m.s. fluctuation is lower at the locations where entrainment from each stream occurs, as shown in figures 20, 22 and 24. Some of the scatter in figures 16 and 17 may also be due to this spanwise variation, which has some $x$-dependence, but there is some uncertainty due to insufficiently long averaging time.

Additional insight into the mean spanwise variations of the concentration can be obtained by comparing simultaneous concentration time histories from both probes. The concentration histories measured by the two probes at $x=9 \mathrm{~cm}, z=0$ and $z=0.64 \mathrm{~cm}$ and at three locations across the layer are presented in figure 25 . At the centre of the layer, $\eta=-0.04$, large concentration fluctuations associated with the primary spanwise vortices dominate the signals. These large fluctuations appear almost simultaneously in time at all levels, indicating the basic two-dimensionality of the large-scale structues. There are, however, differences in the smaller amplitude, higher frequency fluctuations. At the edges, $\eta=0.04$ and -0.09 , there is in general a correlation between large spikes in both signals but their shapes and deviations are not so consistently similar as at the centre of the layer and occasionally one probe shows no evidence of the passage of the vortex seen at the other probe only $0.64 \mathrm{~cm}$ away. This spacing is approximately half the vorticity thickness at this location! Clearly there are large changes in the internal structure of the large, spanwise vortices.

\section{Discussion}

In this investigation we sought to elucidate the vorigin and structure of the secondary streamwise vortices; their relation to and interaction with the primary, spanwise vortices; and their role in the development of the mixing layer. An idealized sketch of our interpretation of their spatial structure is given in figure 15. Unlike earlier interpretations of the streaks, that each streamwise vortex is undirectional (Brown \& Roshko 1974), it is now séen as part of a warped vortex line threading its way up- and downstream between any two adjacent spanwise vortices, thus changing its streamwise directional sign on each pass. This structure is established after the Kelvin-Helmholtz instability has developed into spanwise coherent vortices, possibly as early as the first pairing. The 'wiggle' described by Breidenthal (1981) is its first manifestation.

The streamwise vortex structure originates from an internal instability of the primary vortices. The critical Reynolds number of the instability decreases as the velocity ratio increases, i.e. as the mixing layer approaches a wake. This behaviour suggests that an initial shear-layer profile containing a wake component is more unstable to three-dimensional disturbances. The mean spanwise spacing of the streaks, normalized with the local mean spacing of the primary vortices, is 0.67 , independent of velocity ratio. This is in good agreement with the most-amplified spanwise wavelength found by Pierrehumbert \& Widnall (1982) in their computation of the translative instability of a row of Stuart vortices. The scatter in the measurements is consistent with the fact that the amplification curve is broad. As 
pointed out by Pierrehumbert \& Widnall, this is also consistent with the fact that, once formed, the structure preserves its spanwise scale for some distance downstream, through one or two additional pairings. The initial vortices occur at fixed spanwise locations because they tend to be anchored to upstream spatial non-uniformities no matter how small. However, the spacing does respond to changes in flow conditions; i.e. the existence of the vortices in not $d u e$ to the 'anchors'. Further downstream, after they reform to a larger scale, they are no longer anchored and their spanwise spacing and location fluctuates, as indicated by the photographs made with short and long time exposures.

The proposed warped-vortex-line structure was inferred mainly from a frameby-frame study of a cross-section view film. It is also consistent with the concentration measurements, which show a well-defined spanwise entrainment pattern associated with this structure. Fluid from the high- and low-speed sides is entrained at spanwise positions where the induced velocity of the secondary vortices is directed towards the spanwise vortex cores. The fluids are mixed in the primary vortex core and the resulting mixture is pulled outwards at other spanwise positions where the induced velocity is directed away from the core. The mean and r.m.s. concentration fluctuation are consistent with this pattern. The velocity measurements by Jimenez (1983) show a spanwise variation of constant velocity lines consistent with this interpretation. A direct result of these induced, secondary motions is that the primary spanwise vortices grow between amalgamations. Measurements of this growth in our mixing layers, obtained from high-speed movie films, were reported by Bernal (1981) and, using the same films, by Hernan \& Jimenez (1982). From this it is clear that the secondary structure contributes not only to mixing of passive scalars but also to all components of the Reynolds stress.

The concentration measurements, as well as the pictures, show that the scale of the secondary structures increases with downstream distance. The mean position for the first change has not been precisely determined but both the concentration measurements and the time exposures indicate it to be near the end of the mixing transition. The streaky pattern is still seen on instantaneous pictures downstream of the transition, figure 9, yet it does not come through on time exposures because it varies in time, apparently with the scale of the primary vortices, which now has a broad distribution (Brown \& Roshko 1974). The mean value of the spanwise spacing now scales with the local vorticity thickness, specifically $s / \delta_{\omega}=1.0$ from the concentration measurements at $\rho_{2} / \rho_{1}=7$ and velocity ratio $U_{2} / U_{1}=0.38$. From the picture in figure 9 , using the spanwise spacing of the streaks on the braid at the right of the picture, we find $s / \delta_{\omega}=0.80 \pm 0.14$ for this instantaneous case at uniform density and $U_{2} / U_{1}=0.38$. Jimenez (1983) found, from velocity measurements, $s / \delta_{\omega}=1$ to 1.25 at uniform density and $U_{2}=0$. We conclude that downstream of the mixing transition the mean spacing between adjacent counter-rotating streamwise vortices is given by $s / \delta_{\omega}=1.0$ independent of velocity and density ratio.

It is interesting to compare our results for the spacing of the streamwise vortices with velocity correlation measurements by Wygnanski et al. (1979) and Browand \& Troutt (1980). From the measurements of Wygnanski et al. at the centre of the layer, we estimate a value of $\Delta z / \delta_{\omega} \approx 0.5$ for the location of the minimum cross-correlation coefficient. This value is consistent with our results and indicates that the streamwise vortices are an important contributor to the decay in the spanwise correlation. It should be noted also that those correlation measurements were obtained with a trip wire mounted on the low-speed side of the partition, which generated additional small-scale motions. These apparently did not influence the basic spanwise scale 
associated with the streamwise vortices. The correlation measurements by Wygnanski et al. and Browand \& Troutt at the edge of the layer show a larger spanwise scale. The results of Browand \& Troutt give a characteristic scale of $3 \delta_{\omega}$. This is larger than any scale we have measured that is associated with the streamwise vortex structure; it may be associated with larger-scale spanwise irregularities, such as the helical and other non-uniform pairing events observed by Chandrsuda et al. (1978).

One motivation for this study was to determine the relation between the streamwise vortex structure and the mixing transition. The cross-section pictures obtained in this investigation show that there is a direct contribution of the streamwise vortices to mixing owing to the increased interfacial area in the core of the vortices. This contribution has been evaluated numerically by Lin \& Corcos (1984). As seen in our pictures, figure 11, and in Breidenthal's (1981) pictures, the main contribution to the transition is mixing of unmixed core fluid entrained during the initial two-dimensional development. The streamwise vortices play an indirect role in this process since stretched vorticity along the braids will become part of the spanwise vortex core after amalgamation. This mechanism suggests that the mixing transition should be completed downstream of the three-dimensional instability, after a number of amalgamations. Further, since in the early development the amalgamations can be traced back to the roll-up process and this process depends only weakly on the conditions of the low-speed boundary layer (Ho \& Huang, 1982), we expect the distance to the end of the mixing transition to scale with the thickness of the high-speed boundary layer, as first proposed by Breidenthal (1981). These arguments are also consistent with the results for the mixing transition obtained by Jimenez (1983) and Ho \& Huerre (1984). Thus both, the three-dimensional instability and the amalgamation process play a role in the mixing transition. The instability generates the three-dimensionality, while the amalgamations redistribute it. Although these arguments provide an explanation for the scaling of the mixing transition, the possibility of additional internal instabilities cannot be disregarded.

We note also the fact, mentioned above, that the scale change of the streamwise vortex structure occurs near the end of the mixing transition. The mechanism of this scale change has not been determined. Since the scale of the secondary instability depends on the primary structure, it seems clear that the latter will eventually become too large to sustain the former, which will then die out to be replaced by one of more compatible scale. The latter will tend to fluctuate because the scale of the governing primary structure does. The relationship is made complex by the relatively broad window for the secondary structure. The fundamental problem of the distribution of these scales remains to be clarified. A probabilistic treatment for the primary scales has been given by Bernal (1981).

We wish to thank our colleagues at GALCIT for numerous helpful discussions and other assistance during the course of this investigation, in particular $\mathbf{R} . \mathbf{E}$. Breidenthal, J. E. Broadwell, D. E. Coles and P. E. Dimotakis. The research was sponsored by the Office of Naval Research under contract nos. 8960-1 and N00014-79-C-0365 of Project SQUID and contract no. N00014-C-0260 of the Fluid Dynamics Program. 


\section{REFERENCES}

Bernal, L. P. 1981 The coherent structure of turbulent mixing layers. Ph.D. thesis, California Institute of Technology.

Bradshaw, P. 1966 The effect of initial conditions on the development of a free shear layer. J. Fluid Mech. 26, 225-236.

Breidenthal, R. E. 1978 A chemically reacting turbulent shear layer. Ph.D. thesis, California Institute of Technology.

Breidenthal, R. E. 1979 Chemically reacting, turbulent shear layer. $A I A A J .17,310-311$.

Breidenthal, R. E. 1981 Structure in turbulent mixing layers and wakes using a chemical reaction. J. Fluid Mech. 109, 1-24.

Browand, F. K. \& TroutT, T. R. 1980 A note on spanwise structure in the two-dimensional mixing layer. J. Fluid Mech. 97, 771-781.

Brown, G. L. 1974 The entrainment and large structure in turbulent mixing layers. In Proc. 5th Australasian Conference on Hydraulic.s and Fluid Mechanis, pp. 352-359.

Brown, G. L. \& Rebollo, M. R. 1972 A small, fast-response probe to measure composition of a binary gas mixture. AIAA J. 10, 649-652.

Brown, G. L. \& Roshкo, A. 1971 The effect of density difference on the turbulent mixing layer. AGARD-CP-93, pp. 23-1-23-11.

Brown, G. L. \& Roshкo, A. 1974 On density effects and large structure in turbulent mixing layers. J. Fluid Mech. 64, 775-816.

Chandrsuda, C., Mehta, R. D., Weir, A. D. \& Bradshaw, P. 1978 Effect of free stream turbulence on large structure in turbulent mixing layers. J. F'luid Mech. 85, 693-794.

Dewey, C. F. 1976 Qualitative and quantitative flow field visualization utilizing laser-induced fluorescence. $A G A R D-C P-193$, pp. 17-1-17-7.

Dimotakis, P. E. \& Brown, G. L. 1976 The mixing layer at high Reynolds number: large-structure dynamies and entrainment. J. Fluid Mech. 78, 535-560.

Dimotakis, P. E., Miake-Lye, R. C. \& Papantoniou, D. A. 1983 Structure and dynamies of round turbulent jets. Phys. Fluids. 26, 3285-3192.

Hernan, M. A. \& Jimenez, J. 1982 Computer analysis of a high-speed film of the plane turbulent mixing layer. $J$. Fluid Mech. 119, 323-345.

Ho, C.-M. \& Huang, L. S. 1982 Subarmonics and vortex merging in mixing layers. J. Fluid Mech. $119,445-473$.

Ho, C.-M. \& Huerre, P. 1984 Perturbed free shear layers. Ann. Rev. Fluid Mech. 16, 365-424.

Hussain, A. K. M. F. 1983 Coherent structures and incoherent turbulence. IUTAM Symp. on Turbulence and Chaotic Phenomena in Fluids. Sept. 5-10. Kyoto, Japan.

Jimenez, J. 1983 A spanwise structure in the plane shear layer. J. Fluid Mech. 132, 319-336.

KaTZ, J. \& O'Hern, T. J. 1983 Cavitation in large scale shear flows. ASME paper 83-FE-33.

KonRad, J. H. 1976 An experimental investigation of mixing in two-dimensional turbulent shear flows with applications to diffusion-limited chemical reactions. Ph.D. thesis, California Institute of Technology. Also Project SQUID tec. rep. CIT-8-PU.

Lasheras, J. C., Сho, J. S. \& Maxworthy, T. 1986 On the origin and evolution of streamwise vortical structures in a plane, free shear layer. J. Fluid Mech. (in press).

Lin, S. J. \& Concos, G. M. 1984 The mixing layer: deterministic models of a turbulent flow. Part 3. The effect of plane strain on the dynamics of the streamwise vortices. J. Fluid Mech. 141, 139-178.

Miksad, R. W. 1972 Experiments on the nonlinear stages of free-shear layer transition. $J$. Fluid Mech. 56, 645-719.

Moore, D. W. \& Saffman, P. G. 1975 The density of organized vortices in a turbulent mixing layer. J. Fluid Mech. 64, 465-473.

Patnatk, P. C., Sherman, F.S. \& Corcos, G. M. 1976 A numerical simulation of Kelvin-Helmholtz waves of finite amplitude. J. Fluid Mech. 73, 215-240.

Pierrehumbert, R. T. \& Widnald, S. E. 1982 The two- and three-dimensional instabilities of a spatially periodic shear layer. J. Fluid Mech. 114, 59-82. 
Roshko, A. 1976 Structure of turbulent shear flows; a new look. AIAA J. 14, 1349-1357.

Winant, C. D. \& Browand, F. K. 1974 Vortex pairing: the mechanism of turbulent mixing layer growth at moderate Reynolds number. J. Fluid Mech. 63, 237-255.

Wygnanski, I., Oster, D., Fiedler, H. \& Dziomba, B. 1979 On the perseverance of a quasi-two-dimensional eddy-structure in a turbulent mixing layer. J. Fluid Mech. 93, 325-335. 